\title{
Transform Methods for the Reduction of the Peak to Average Power Ratio for the OFDM Signal
}

\author{
Rajendra Kumar and Vuttipol Santitewagul \\ Department of Electrical Engineering, California State University, Long Beach, CA 90840, USA \\ Correspondence should be addressed to Rajendra Kumar; rajendra.kumar@csulb.edu
}

Received 26 July 2016; Revised 5 September 2016; Accepted 15 September 2016; Published 12 January 2017

Academic Editor: Michael McGuire

Copyright (c) 2017 R. Kumar and V. Santitewagul. This is an open access article distributed under the Creative Commons Attribution License, which permits unrestricted use, distribution, and reproduction in any medium, provided the original work is properly cited.

\begin{abstract}
The paper presents multitransform OFDM-OP system for an effective PAPR (peak to average power ratio) reduction that has a reasonable computational requirement, does not introduce any distortion, needs relatively insignificant decrease in the bandwidth efficiency, and provides a PAPR very close to that for the single carrier modulation systems thus effectively eliminating any PAPR penalty incurred by the multicarrier OFDM system. The PAPR system consists of a bank of multiple orthonormal transforms and a minimum PAPR evaluation unit for finding the optimum transform index. The paper also presents a hybrid OFDM-OP-DSI system comprised of the multiple transforms and a novel dummy symbol insertion. The PAPR reduction performance of the presented systems is compared with those of the various other transform techniques of the literature. Various simulations on the presented systems show that these can achieve a PAPR that is very close to that of a single carrier system for QAM modulation with the order of modulation selected to be 16,64 , and 256 .
\end{abstract}

\section{Introduction}

Broadband wireless systems are in a rapidly evolutionary phase in terms of development of various technologies, development of various applications, deployment of various services, and generation of many important standards in the field [1-28]. Orthogonal Frequency Division Multiple Accessing (OFDM) techniques offer efficient bandwidth utilization and provide many other advantages such as some immunity against the distortion due to the multipath propagation environment. Therefore, the OFDM techniques have been adapted in many wireless communication and sensor standards, such as the Worldwide Interoperability for Microwave ACCESS (WiMAX), digital audio broadcasting (DAB), digital video broadcasting-terrestrial (DVB-T), and Long Term Evolution (LTE).

However, the use of a relatively large number of carriers used in the OFDM signal results in a relatively high peak to average power ratio resulting in a much reduced radio frequency (RF) power amplifier efficiency and distortion due to the amplifier nonlinearity. In order to keep the distortion to some specified limit, the output RF power is backed off from the maximum available power at the amplifier output. In addition to the reduced output power, the output backoff concurrently also results in the DC to RF power conversion efficiency. A detailed analysis of the distortion effects of the nonlinear power amplifier and some of the mitigating techniques are presented, for example, in $[8,9]$ and the references therein. Another problem arising due to distortion caused by the amplifier is the spreading of the spectrum of the OFDM signal outside the allocated band [10].

Thus there has been strong motivation to come up with techniques that can reduce the peak to average power ratio of the OFDM signal without causing any distortion in the process of transformation, or losing in terms of bandwidth or other efficiency measures. The paper presents multitransform OFDM-OP system for an effective PAPR (peak to average power ratio) reduction that has a reasonable computational requirement, does not introduce any distortion, needs relatively insignificant decrease in the bandwidth efficiency, and provides a PAPR very close to that for the single carrier modulation systems thus effectively eliminating any PAPR penalty incurred by the multicarrier OFDM system. 


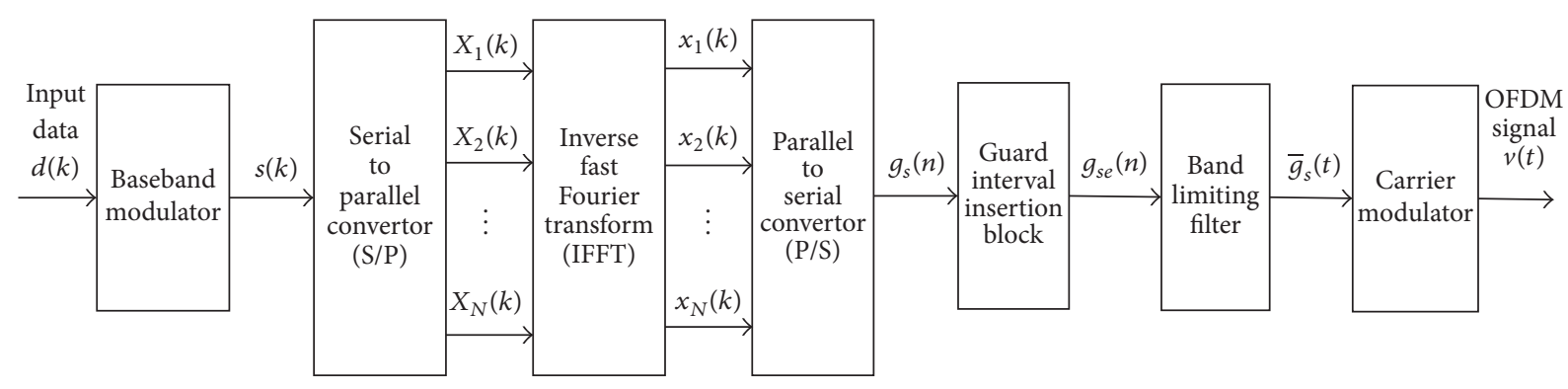

FIGURE 1: Block diagram of the OFDM system.

The contents of the paper are organized as follows. Section 2 of the paper presents a brief introduction to the OFDM system along with various notations used in the paper. Section 3 provides a brief review of the various peak to average power ratio (PAPR) reduction techniques in the published literature. Section 4 presents multitransform systems and methods recently invented by the first author of the paper and taught in US Patent 8,995,542, March 2015 [11], for an effective PAPR reduction which have a reasonable computational requirement, do not introduce any distortion, need relatively insignificant decrease in the bandwidth efficiency, and provide PAPR very close to that for the single carrier modulation systems thus effectively eliminating any PAPR penalty incurred by the multicarrier OFDM system. Section 5 presents simulation results on the performance of the various PAPR reduction techniques. Section 6 presents some concluding remarks.

\section{OFDM System}

An OFDM-modulated signal consists of the parallel transmission of several signals that are modulated at different carrier frequencies evenly spaced by $\Delta f$ [1-7]. The complex valued input symbol sequence $\{s(j)\}$ is split into $N$ subsequences $\left\{s_{m}(k)\right\}$ with $s_{m}(k)=s(n), n=k N+m, m=$ $0,1, \ldots, N-1 ; k=0,1,2, \ldots$. The symbol subsequence $\left\{s_{m}(k)\right\}$ modulates a corresponding subcarrier at frequency $f_{m}$ for $m=0,1, \ldots, N-1$. Thus the time sampled version of the complex envelope $g_{s}(n)$ of the modulated signal is given by (1) wherein the sampling period $T_{S}=T_{0} / N$ with $T_{0}$ denoting the symbol period for the subsequence $\left\{s_{m}(j)\right\}$.

$$
\begin{aligned}
g_{s}(n+k N)=\left\{\frac{1}{\sqrt{N}} \sum_{m=0}^{N-1} s_{m}(k) \exp \left[\frac{j 2 \pi m n}{N}\right]\right\} ; \\
n=0,1, \ldots, N-1 ; k=0,1, \ldots
\end{aligned}
$$

The consecutive $N$ samples of $g_{s}(n)$ constitute an OFDM symbol and according to (1) the samples during the $k$ th OFDM symbol may be obtained by an $N$ point IFFT (inverse fast Fourier transform) of the consecutive $N$ symbols in the symbol sequence $\{s(j)\}$ or the $k$ th symbols in the symbol subsequences $\left\{s_{m}(k)\right\}, m=0,1, \ldots,(N-1)$. In the multiple access application of OFDM the symbol subsequence $\left\{s_{m}(k)\right\}$ may be the symbol sequences generated for the $N$ multiple access users rather than subsequences of a single user symbol sequence. The OFDM signal has a guard interval of length $T_{G}$ for each OFDM symbol to mitigate the intersymbol interference. The sample values during any guard interval are obtained by the periodic extension of the subsequent $N$ sample values of $g_{s}(n)$. The transmitted radio frequency $(\mathrm{RF})$ OFDM signal $v(t)$ is given by

$$
v(t)=\operatorname{Re}\left\{\bar{g}_{s}(t) \exp \left[j 2 \pi f_{c} t\right]\right\},
$$

where $f_{c}$ denotes the carrier frequency and $\bar{g}_{s}(t)$ denotes the continuous time signal obtained by interpolation of the sampled signal $g_{s}(n)$ using, for example, 0 th order hold. The signal $g_{s}(n)$ may also be band limited by a band limiting filter such as the square root raised cosine filter in generating the analog signal $\bar{g}_{s}(t)$.

Figure 1 shows the block diagram of the OFDM system. Referring to Figure 1, the data $d(k)$ that may be a binary stream is inputted to the baseband modulator block that modulates the input data according to a modulation scheme that is selected to be the QAM modulation. The results of the paper will apply equally well to various other modulation techniques such as MPSK or MASK modulation schemes. The complex baseband signal $s(k), k=0,1, \ldots$, is inputted to the serial to parallel converter with the output given by the OFDM modulation symbol vector $X(k)=$ $\left[\begin{array}{llll}X_{1}(k) & X_{2}(k) & \cdots & X_{N}(k)\end{array}\right]^{T}$ where $X_{m}(k)=s_{m}(k)$, or $X_{m}(k)=s(n), n=k N+m ; m=0,1, \ldots, N-1$; $k=0,1, \ldots$. The inverse fast Fourier transform (IFFT) block provides the inverse Fourier transform of $X(k)$ providing the OFDM modulated signal vector $x(k)$ of dimension $N$ also referred to as the OFDM frame at the output that is inputted to the parallel to serial converter block. The parallel to serial converter block concatenates the components $x_{m}(k)$ of the vector $x(k)$ providing the baseband OFDM signal $g_{s}(n)$, $n=k N+m$ given by (1). The complex baseband OFDM signal $g_{s}(n)$ is inputted into the guard band insert block for extending the OFDM signal duration by the guard interval $T_{G}$ by a periodic extension of the signal $g_{s}(n)$. The OFDM baseband signal with a guard interval denoted by $g_{s e}(n)$ is inputted to a band limiting filter that may be, for example, a square root raised cosine filter and may include a digital to analog converter providing the filtered complex baseband OFDM signal $\bar{g}_{s}(t)$ that modulates a carrier signal providing the bandpass OFDM signal $v(t)$ given by (2). This paper is focused upon the subsystem of the OFDM system that 
generates the complex baseband OFDM signal $g_{s}(n)$ from the input data $d(k)$.

The peak to average power ratio PAPR is defined as

$$
\mathrm{PAPR}=10 \log \left\{\frac{\max _{t}\left|\bar{g}_{s}(t)\right|^{2}}{E\left[\left|\bar{g}_{s}(t)\right|^{2}\right]}\right\} .
$$

In (3) $E$ denotes the expected value. As $\bar{g}_{s}(t)$ is a random process, PAPR is a random variable with some probability distribution function or equivalently in terms of a cumulative distribution function (CDF) or equivalently the complementary cumulative distribution function (CCDF) $G(\gamma)$ that is function of the real variable $\gamma$ given by $G(\gamma) \equiv \operatorname{Prob}\{$ PAPR $>$ $\gamma$ \}. It is of interest to minimize the $G(\gamma)$ for any specified value of $\gamma$ by some possible invertible transform of the signal $\bar{g}_{s}(t)$. In practice the PAPR is defined in terms of the modulated signal vector $x(k)$ as

$$
\mathrm{PAPR} \cong \frac{\max _{n}\left|x_{n}(k)\right|^{2}}{\|x(k)\|^{2} / N}
$$

and the CCDF is defined in terms of the time samples of the PAPR in (4).

\section{Techniques for the Reduction of the Peak to Average Power Ratio}

There have been several solutions proposed in the literature to reduce the peak to average power ratio of the OFDM signal. One such method is the clipping method [12] wherein the signal above a certain specified value is clipped. This is similar to the clipping by the amplifier and thus introduces distortion; however, clipping and filtering the signal before inputting to the RF amplifier may mitigate the problem of spectrum spreading that is encountered by the clipping caused by the amplifier.

In another PAPR reduction method proposed in [13] and termed the selective mapping (SLM) method consists of forming $Q$ vectors $P^{q}, q=1,2, \ldots, Q$, with $Q$ an integer being formed with the $i$ th element of the vector $P^{q}$ selected equal to $P_{i}^{q}=\exp \left[j \varphi_{i}^{q}\right] ; j=\sqrt{-1}, i=0,1, \ldots, N-1$. The phase $\varphi_{i}^{q}$ is selected in a random manner with a uniform probability density function over the interval $[0,2 \pi]$. The set of vectors thus formed is made known to the receiver in advance. For any time $k$, the OFDM modulation symbol vector $X(k)$ is component-wise multiplied by each of the $Q$ vectors $P^{q}$ resulting in the modified vector $X^{q}(k), q=$ $1,2, \ldots, Q$. This follows evaluation the inverse fast Fourier transform (IFFT) $x^{q}(k)$ of $X^{q}(k)$ and computing the peak to average power ratio of the OFDM modulation signal vector $x^{q}(k)$ for $q=1,2, \ldots, Q$. The vector $x^{q}(k)$ with the minimum PAPR is selected for transmission with the corresponding index $q_{0}$ made available to the receiver as a side information. The result presented in [13] for the case of $N=128$ and $Q=4$ and QPSK modulation shows an improvement of about $3 \mathrm{~dB}$ at a PAPR value corresponding to the complementary probability distribution function (CPDF) value of $10^{-3}$.
In the partial transmit sequence (PTS) method proposed in [14], the set of indices 0 through $N-1$ is partitioned into $V$ disjoint subsets $S_{v}, v=1,2, \ldots, V$, wherein each of the $V$ subsets has $(N / V)$ indices. For $v$ equal to 1 through $V$, a vector $X^{v}(k)$ of length $N$ is obtained with all its elements equal to 0 except the ones with indices in the subset $S_{v}$ that are selected to be equal to the corresponding elements of the vector $X(k)$ resulting in $X(k)=\sum_{v=1}^{V} X^{v}(k)$. Each of the $V$ vectors is inverse Fourier transformed using the IFFT providing the $V$ signal vectors $x^{v}(k)=\mathscr{F}^{-1}\left\{X^{v}(K)\right\}$ wherein $\mathscr{F}^{-1}$ denotes the inverse Fourier transform. The signal vectors are multiplied by the complex scalars $\exp \left[j \varphi^{v}(k)\right]$ with $\varphi^{v}$ selected randomly and are uniformly distributed over the interval $(0,2 \pi)$. The weighted signal vectors are summed and the PAPR of the resulting sum is computed. The PAPR is minimized over the selection of the scalars $\exp \left[j \varphi^{v}(k)\right]$ and the result of such a minimization is selected for transmission. The selected coefficients are provided to the receiver as a side information. The simulation results in [14] show that for the case of $V=Q$ and for QPSK modulation the PTS scheme provides a better performance compared to that of the SLM method.

A dummy sequence insertion (DSI) method of the PAPR reduction has been proposed in [16]. In the DSI method, the vector $X(k)$ is comprised of $N_{I}$ modulation symbols and $N_{D}=\left(N-N_{I}\right)$ dummy symbols resulting in $X(k)=$ $\left[\begin{array}{ll}X^{I T}(k) & X^{D T}(k)\end{array}\right]^{T}$ wherein $T$ denotes the matrix transpose, and $X^{I}(k)$ and $X^{D}(k)$ are the vectors of length of $N_{I}$ and $N_{D}$ and comprised of the modulation symbols and dummy symbols, respectively. The DSI method results in a reduction of the bandwidth efficiency by a factor of $\left(N_{I} / N\right)$; however, it does not require any side information. The selection of the dummy sequence is comprised of an initial step and a recursive step that modifies the dummy sequence until the PAPR of $x(k)=\mathscr{F}^{-1}\{X(K)\}$ is below a threshold or the number of recursions exceeds some maximum permissible number of recursions. Four different methods for the selection of the dummy sequence are suggested in [16]. In the first method, the dummy sequence is comprised of a complementary sequence [16] with different complementary sequences selected in the recursive step. In another method, the initial dummy sequence is selected to be an all 0 or an all 1 sequence, with the recursion step comprised of sequentially flipping the dummy sequence bits until the PAPR below the threshold value is achieved or the number of recursions exceeds a specified limit.

In the method of selective scrambling proposed in [17], the message bit sequence is scrambled by each of the four maximal lengths or $\mathrm{m}$-sequences that are not cyclically shifted versions of each other, with the two bits representing the index $q$ of the $\mathrm{m}$-sequence appended to the scrambled sequence. The scrambled sequences are modulated into QPSK symbols resulting in the OFDM modulation symbol vectors $X^{q}(k)$ which are inverse Fourier transformed resulting in the OFDM modulated signal vectors $x^{q}(k)$ for $q=1,2,3$ and 4 . The vector among the 4 vectors $x^{q}(k)$ with a minimum PAPR is selected for transmission. This method is very similar to the SMI method with the difference that 
it is the bit sequence that is scrambled instead of the QPSK modulation symbol sequence in the SMI method.

In the block coding schemes for the PAPR reduction, the OFDM modulation symbol vector $X(k)$ is transformed using one of the block error correction codes [18-20]. For example, the use of complementary sequence codes is presented in [18]. While the use of the block error correction codes to reduce the PAPR while simultaneously achieving the error correction capability of the code is clearly very appealing, however, the presently studied methods based on block error correction codes may require relatively very low rate codes resulting in relatively poor bandwidth efficiency at relatively high number of carriers. To quote from [18], the authors state that, "The major drawback of the peak power controlling block coding scheme is that the coding rate is inversely proportional to the number of OFDM subcarriers. For example, for permissible PAPR of $6 \mathrm{~dB}$, the coding rate of the 128 -subcarrier system becomes $7 / 64=0.11$, which clearly seems quite impractical for many applications." Similar results appear in $[19,20]$.

In the precoding techniques proposed in [21-23] the OFDM modulation symbol vector $X(k)$ is premultiplied by an orthogonal matrix $P$ resulting in the transformed symbol vector $X^{p}(k)=P X(k)$. The inverse Fourier transform of the transformed symbol vector provides the modulated signal vector $x(k)$ for the transmission. The precoding matrix $P$ is signal independent and is known to the receiver. The orthogonal transform is selected to be the discrete Hartley transform (DHT) in [21], discrete cosine transform in [22], and the Walsh-Hadamard transform (WHT) in [23], respectively. The precoding techniques are very attractive as these do not introduce any distortion unlike the clipping based methods, do not result in any reduction in the bandwidth efficiency as is the case with block coding methods, and are relatively simple in implementation. In [23] the precoding technique is combined with the SLM and DSI methods for providing further improvement in their PAPR reduction capability at the expense of some additional complexity of implementation and some reduction the bandwidth efficiency. It is shown in this paper that there is a scope for further improving their PAPR reduction capability while maintaining their desirable characteristics.

\section{Multitransform Method for PAPR Reduction}

This section in its entirety describes the multitransform systems and methods for the reduction of the peak to average power ratio recently invented by the first author of this paper and taught in and protected by US Patent $8,995,542$, March 31, 2015 [11]. Figure 2 shows the block diagram of the proposed multitransform method. As shown in the figure, the modulation symbol vector $X(k)$ is transformed by a number $N_{T}$ of transforms providing $N_{T}$ transformed symbol vectors $X^{1}(k), X^{2}(k), \ldots, X^{N_{T}}(k)$ with

$$
X^{n}(k)=P^{n} X(k) ; \quad n=1,2, \ldots, N_{T} ; k=0,1,2, \ldots
$$

The IFFT blocks in Figure 2 provide the IFFT of the transformed symbol vectors $X^{n}(k)$ denoted by $x^{n}(k)$ for $n=$ $1,2, \ldots, N_{T}$. The input selector block in Figure 2 selects the one out of the $N_{T}$ input vectors $x^{n}(k)$ with the lowest PAPR computed according to (4). The output of the input selector block is inputted to the parallel to serial converter that generates the serial sample sequence $g_{s}(n)$ at the output.

In (5) $P^{n}$ for $n=1,2, \ldots, N$ are some conveniently selected $N \times N$ nonsingular matrices. For example, with $N_{T}=$ 4 , the 4 matrices that are selected are the identity matrix $I_{N}$ corresponding to no transform, the Walsh-Hadamard transform (WHT) matrix $P^{W}$, the discrete cosine transform (DCT) matrix $P^{C}$, and the discrete Hartley transform (DHT) matrix $P^{H}$. The three transform matrices are given in terms of their $(m, n)$ th element, $m, n=1,2, \ldots, N$, by

$$
\begin{aligned}
P_{m, n}^{H} & =\frac{1}{\sqrt{N}}\left\{\cos \left[\frac{2 \pi(m-1)(n-1)}{N}\right]\right. \\
+ & \left.\sin \left[\frac{2 \pi(m-1)(n-1)}{N}\right]\right\} \\
P_{m, n}^{C} & =\sqrt{\frac{2}{N}} \cos \left[\frac{\pi(m-0.5)(n-0.5)}{N}\right]
\end{aligned}
$$

with the Walsh-Hadamard transform matrix $P^{W}$ with its elements equal to +1 or -1 defined recursively in terms of the matrix $W$ as

$$
\begin{aligned}
& W_{2^{m}}=\left[\begin{array}{ll}
W_{2^{m-1}} & W_{2^{m-1}} \\
W_{2^{m-1}} & -W_{2^{m-1}}
\end{array}\right] ; \\
& W_{2}=\left[\begin{array}{cc}
1 & 1 \\
1 & -1
\end{array}\right] ; \\
& P^{W}=\frac{1}{\sqrt{N}} W_{2^{m_{0}}} ; \quad N=2^{m_{0}} .
\end{aligned}
$$

In the same manner, the inverse Fourier transform may be expressed in terms of the transform matrix $P^{F}$ given by

$$
P_{m, n}^{F}=\frac{1}{\sqrt{N}} \exp \left[\frac{2 \pi j(m-1)(n-1)}{N}\right] ; \quad j=\sqrt{-1} .
$$

The use of scalar $1 / \sqrt{N}$ in (6)-(9) introduced for the sake of clarity makes these matrices orthonormal with $P P^{H}=I_{N}$ or $P^{-1}=P^{H}$ for any of the transform matrices $P$ in (6)-(9) with the superscript $H$ denoting the matrix Hermitian transpose and $I_{N}$ denoting the $N \times N$ identity matrix. However, the scale factor $1 / \sqrt{N}$ in (6)-(9) may be eliminated without introducing any changes in the performance results for the OFDM system. Due to symmetry the matrices $P^{H}$, $P^{W}$, and $P^{C}$ are also unitary with $P^{-1}=P$. The use of these orthogonal matrices permits the use of fast transform techniques permitting the matrix vector multiplication in order $\operatorname{Nlog}_{2}(N)$ operation instead of requiring order $N^{2}$ operations for obtaining the transformed symbol vector 


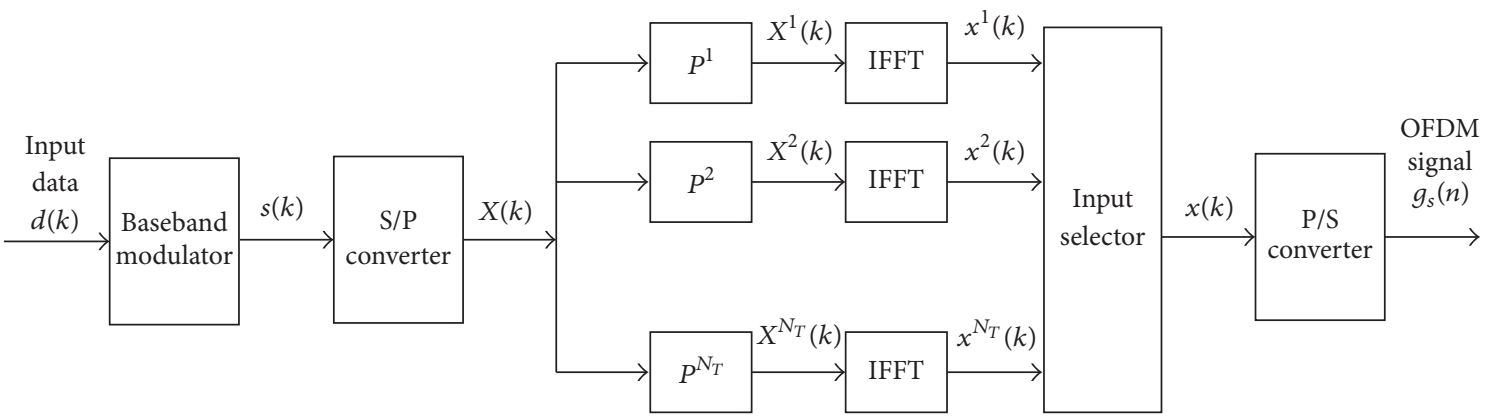

FIgURE 2: Multitransform OFDM system for PAPR reduction.

$X^{n}(k)$. In fact, the number of operations can be further reduced by exploiting the relationships between various transforms. In particular they may be related to the Fourier transform. For example, for a real valued sequence $X^{R}(k)$ its DHT transform may be obtained by [26, 27]

$$
\begin{aligned}
& \operatorname{DHT}\left\{X^{R}(k)\right\}=\operatorname{Re}\left\{(1-j) \mathscr{F}^{-1}\left[X^{R}(k)\right]\right\} ; \\
& j=\sqrt{-1} .
\end{aligned}
$$

In (10) $\mathscr{F}^{-1}$ denotes the inverse Fourier transform, and $\operatorname{Re}(z)$ for any complex quantity $z$ denotes the real part of $z$. With $X(k)=X^{R}(k)+j X^{I}(k)$, its DHT transform may be evaluated as

$$
\begin{aligned}
\operatorname{DHT}\{X(k)\}= & \operatorname{Re}\left\{(1-j) \mathscr{F}^{-1}\left[X^{R}(k)\right]\right\} \\
& +j \operatorname{Re}\left\{(1-j) \mathscr{F}^{-1}\left[X^{I}(k)\right]\right\} .
\end{aligned}
$$

Thus computing the IFFT of $X^{R}(k)$ and $X^{I}(k)$ separately permits a direct computation of the DHT form (11) requiring only order $N$ operations. Of course, the IFFT of $X(k)$ is given by

$$
\mathscr{F}^{-1}\{X(k)\}=\mathscr{F}^{-1}\left[X^{R}(k)\right]+j \mathscr{F}^{-1}\left[X^{I}(k)\right] .
$$

The computation of IFFT of $X(k)$ from (12) does not require any more computations compared to directly computing the IFFT of $X(k)$. In the same manner the WHT of $X^{R}(k)$ may be computed in terms of the FFT or IFFT of $X^{R}(k)$. For example, [29] describes a method of computing the Fourier transform of a real sequence in terms of its Walsh-Hadamard transform. The relationship given in [29] can be more easily used for the computation of WHT from the IFFT. For example, equation (6) of [29] relates the WHT to the IFFT for the case of $N=8$ requiring only 10 real multiplications equivalent to less than 3 complex multiplications. Similar computations are given in [29] for more general value of $N$; details are not presented here. Thus for the case of $N_{T}=4$, the order of transforms and IFFT may be performed as shown in Figure 3.

Another example of the multitransform method consists of the use of $N_{T}>4$ transform matrices including the $I$,

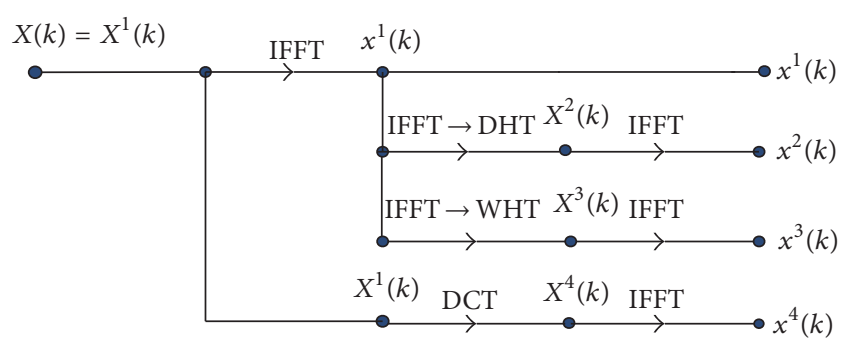

FIGURE 3: Order of transform computations $\left(N_{T}=4\right)$.

$P^{H}, P^{W}$, and $P^{C}$ and the possible products of these matrices such as $P^{H} P^{C}$. Table 2 lists some of these transform matrices with their associated indices that are referred to in the simulation results of the next section. In the computation of the transformed vectors $x^{n}(k)$, Figure 3 may be used for the minimization of the computational requirements as for the case of $N_{T}=4$. For any pair orthonormal matrices $P^{1}$ and $P^{2}$ one obtains

$$
\left(P^{1} P^{2}\right)^{-1}=\left(P^{2}\right)^{-1}\left(P^{1}\right)^{-1}=P^{2 H} P^{1 H}=\left(P^{1} P^{2}\right)^{H} .
$$

Thus the product of any two orthonormal matrices is also orthonormal and all of the $N_{T}$ transforms selected for the PAPR reduction are orthonormal transforms. The set of all possible $(N \times N)$ orthonormal matrices forms a group under matrix multiplication.

4.1. Multitransform Method with Dummy Sequence Insertion. The multiple transform method may be combined with the dummy insertion method resulting in the OFDM-OP-DSI method for the PAPR reduction wherein OP refers to the optimum transform. In this method the OFDM symbol vector $X(k)$ is comprised of $N_{D}$ dummy symbols and $N_{I}=$ $N-N_{D}$ information symbols. The dummy symbols may correspond to $N_{D}$ randomly selected but fixed indices of the vector $X(k)$; for example, the first $N_{D}$ elements may be the dummy symbols. Figure 4 shows the block diagram of the direct implementation of the OFDM-OP-DSI method. In Figure $4 X^{I}(k)$ is the vector of length $N$ with $N_{I}$ elements equal to the information symbols and $N_{D}=N-N_{I}$ elements 


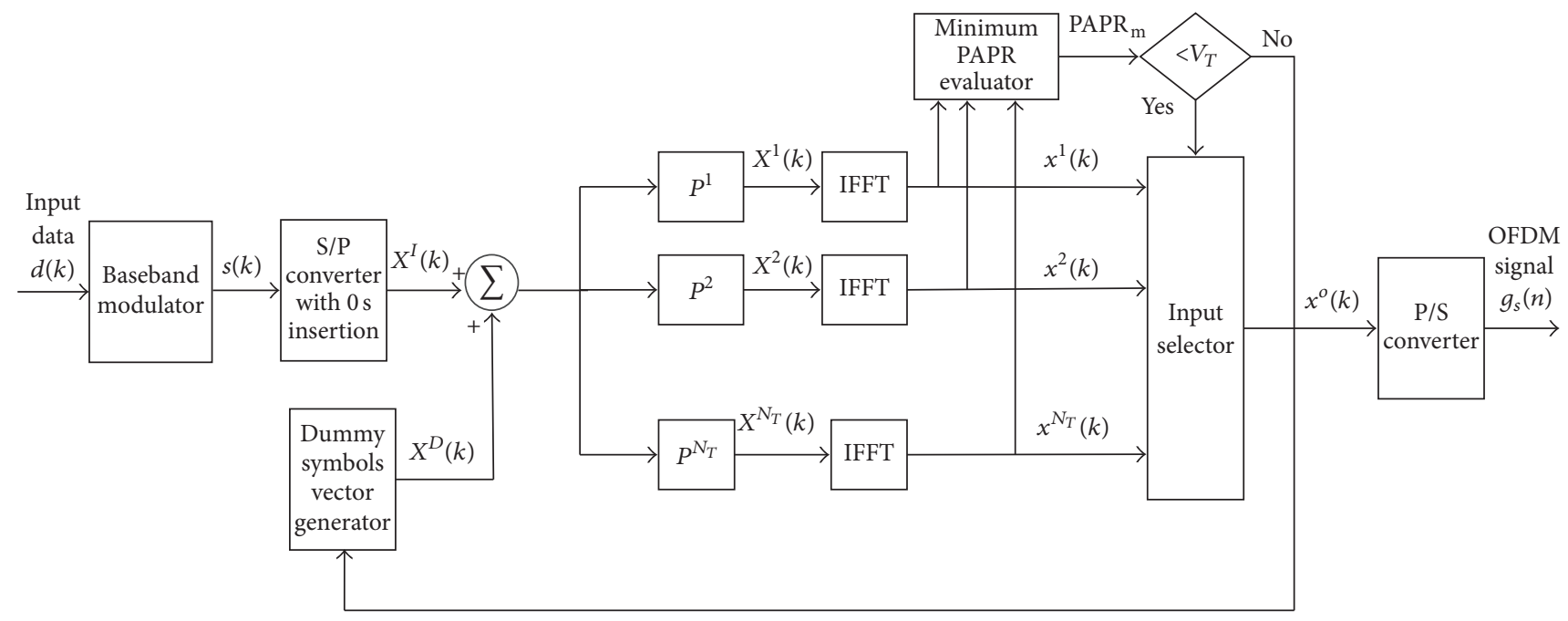

FIGURE 4: Multitransform-DSI OFDM system for PAPR reduction.

equal to 0 with the set $\delta_{I}$ comprised of their indices. The elements of the length $N$ vector $X^{D}(k)$ with indices the set $\mathcal{S}_{I}$ are equal to the dummy symbols with the other $N_{I}=N-N_{D}$ elements set equal to 0 . As shown in the figure, the vector $X(k)=X^{I}(k)+X^{D}(k)$ is inputted to the $N_{T}$ transform blocks that multiply the vector $X(k)$ by the matrices $P^{n}$ providing the transformed vectors $X^{n}(k), n=1,2, \ldots, N_{T}$, at their outputs.

The transformed vectors are inputted to the IFFT blocks providing the inverse Fourier transforms $x^{n}(k)$ of the transformed vectors $X^{n}(k)$ to the input selector block that selects the input with the lowest PAPR from the $N_{T}$ inputs. As shown in the figure, the transformed OFDM signal vectors $x^{n}(k)$ are inputted to the minimum PAPR evaluator block that evaluates the minimum of the PAPRs of the $N_{T}$ OFDM signal vectors $x^{n}(k)$ and provides the result $\mathrm{PAPR}_{\mathrm{m}}$ to the decision block. The decision block compares the $\mathrm{PAPR}_{\mathrm{m}}$ with a threshold $V_{T}$. If the threshold condition is satisfied, the input selector block selects the input with the minimum PAPR and inputs the selected vector $x(k)$ to the vector to serial converter that outputs the OFDM complex baseband signal. If the threshold condition is not satisfied, the process is repeated with a different selection of the dummy symbols. The selection of the dummy symbols either may be performed in a predetermined sequence or may be based on a random selection strategy. Figure 12 shows a histogram of the optimum dummy symbol selection for an example of 64 QAM modulation. In the multitransform-DSI method based on a threshold, the sequencing of the dummy symbol selection may be performed according to the histogram as shown in Figure 12 .

The computational requirements of the optimum transform-DSI method can be significantly reduced by an appropriate organization of the computations. The transformed signal vector $x^{n}(k)$ may be expressed as

$$
x^{n}(k)=P^{F} P^{n} X(k)=P^{F} P^{n} X^{I}(k)+P^{F} P^{n} X^{D}(k) .
$$

With $j_{1}, j_{2}, \ldots, j_{N_{D}}$ denoting the indices of the vector $X(k)$ corresponding to the dummy symbols, the vector $x^{n}(k)$ may be expressed as

$$
\begin{aligned}
x^{n}(k) & =P^{F} P^{n} X^{I}(k)+P^{F} P_{S}^{n} y_{D}(k) \\
& =P^{F} P^{n} X^{I}(k)+P_{D}^{n} y_{D}(k) .
\end{aligned}
$$

In (14b) $y_{D}$ is the vector of length $N_{D}$ with its elements equal to the dummy symbols and $P_{S}^{n}$ is the $\left(N \times N_{D}\right)$ submatrix of $P^{n}$ comprised of the $N_{D}$ columns of the matrix $P^{n}$ with indices $j_{1}, j_{2}, \ldots, j_{N_{D}}$; that is, the matrix $P_{S}^{n}$ is given by

$$
P_{S}^{n}=\left[\begin{array}{llll}
P_{j_{1}}^{n} & P_{j_{2}}^{n} & \cdots & P_{j_{N_{T}}}^{n}
\end{array}\right]
$$

and $P_{D}^{n}$ denotes the matrix with its columns equal to the inverse Fourier transforms of $P_{j}^{n} ; i=1,2, \ldots, N_{D}$. In (15) $P_{j}^{n}$ denotes the $j$ th column of the matrix $P^{n}$ for any integer $j$. In the specific case of $N_{D}=1$ considered in the simulations presented in the paper, the signal vector $x^{n}(k)$ may be expressed as

$$
x^{n}(k)=P^{F} P^{n} X^{I}(k)+p^{n} s_{D}(k) .
$$

In $(16) s_{D}(k)$ denotes the dummy symbol and $p^{n}$ is a column vector given by the Fourier transform of the $j$ th column of $P^{n}$ wherein $j$ is the index of the dummy symbol in the OFDM symbol vector $X(k)$ that may be precomputed and stored for use in the real time application. Thus the change of the dummy symbol requires just the multiplication of a fixed vector $p^{n}$ by the selected symbol for the selected transform matrix $P^{n}$, rather than requiring an $N$ point IFFT for each symbol selection and each $n$ resulting in considerable saving in the computational requirements.

Figure 5 shows the block diagram of the computationally efficient OFDM-OP-DSI method for the case of $N_{D}=1$. In Figure $5 X^{I}(k)$ vector of length $N$ with $N_{I}$ elements equal to the information symbols and $N_{D}=N-N_{I}$ elements 


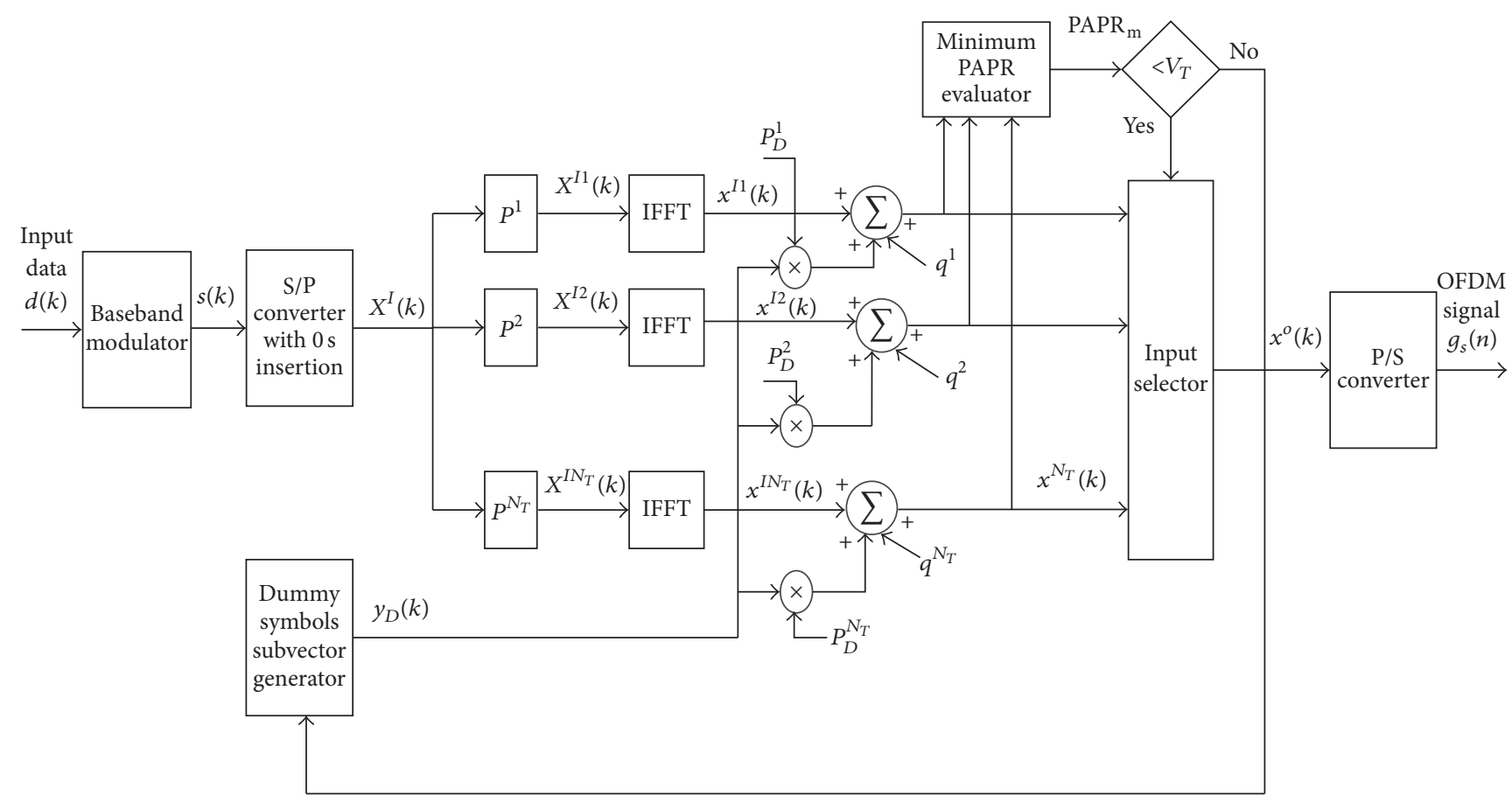

FIGURE 5: Computationally efficient multitransform-DSI OFDM system for PAPR reduction.

equal to 0 with their indices in the set $\mathcal{S}_{I}$ is inputted to the $N_{T}$ transform blocks providing the transformed outputs $X^{I, n}(k)$ for $n=1,2, \ldots, N_{T}$. The transformed outputs $X^{I, n}(k)$ are inputted to the IFFT blocks providing the transformed OFDM information signal vectors $x^{I, n}(k)$ at the outputs. As shown in the figure, the dummy symbol vector $y_{D}(k)$ at the output of the dummy symbol selector is multiplied by the vectors $P_{D}^{1}, P_{D}^{2}, \ldots, P_{D}^{N_{T}}$ with the result added to the respective transformed OFDM information signal vectors $x^{I, n}(k)$ resulting in the transformed OFDM signal vectors $x^{n}(k) ; n=1,2, \ldots, N_{T}$.

As shown in Figure 5, the transformed OFDM signal vectors $x^{n}(k)$ are inputted to the minimum PAPR evaluator block that evaluates the minimum of the PAPRs of the $N_{T}$ OFDM signal vectors $x^{n}(k)$ and provides the result PAPR $\mathrm{m}_{\mathrm{m}}$ to the decision block. The decision block compares the $\mathrm{PAPR}_{\mathrm{m}}$ with a threshold $V_{T}$. If the threshold condition is satisfied, the input selector block selects the input with the minimum PAPR and inputs the selected vector $x(k)$ to the vector to serial converter that outputs the OFDM complex baseband signal. If the threshold condition is not satisfied, the process is repeated with a different selection of the dummy symbols.

The information about the selected transform can be imbedded into the OFDM signal by using one or more symbols of the OFDM frame for this purpose. For the case wherein the order of modulation $M$ is greater than or equal to 64 and the number of transforms $N_{T}$ is less than 16 as proposed in the paper, one symbol is adequate for carrying this information. In fact for $M \geq 64$, significant error correction coding on the transform index may be used to protect against error. Using one symbol for carrying this side information, the number of zeros in the vector $X^{I}(k)$ is made equal to $\left(N_{D}+1\right)$. Assuming that the side information is contained in the first element of the OFDM modulation symbol vector $X(k)$, the vector to be added to the modified information signal vector $x^{I, n}(k)$ in Figure 5 is given by

$$
q^{n}=P^{F} P_{1}^{n} s_{i}^{n}
$$

In (17) $P_{1}^{n}$ denotes the first column of the transform matrix $P^{n}$, and $s_{i}^{n}$ is the symbol containing the index $n$ of the transform in a possibly coded form. As shown in Figure 5, the fixed vector (not a function of time $k$ ) $q^{n}$ is added as a bias to the modified information signal vector $x^{I, n}(k)$ resulting in the modified modulation signal vector $x^{n}(k)$ given by (18) for the case of $N_{D}=1$.

$$
x^{n}(k)=x^{I, n}(k)+p^{n}(k)+q^{n},
$$

where in (18) the first term on the right hand side is dependent upon the information symbols $s_{k}$ at the output of the baseband modulator, the second term $p^{n}(k)$ is dependent upon the dummy symbols selected, and the last term $q^{n}$ that is independent of $k$ provides the side information about the index of the transform.

The selection of the indexing symbol $s_{i}^{n}$ used to encode the transform index $n$ for $n=1,2, \ldots, N_{T}$ is made from the signal constellation diagram of the complex baseband signal $s(k)$ so as to minimize the probability of error in the detection of $n$ at the OFDM receiver. For example, for the case of 64 QAM modulation with the signal constellation diagram shown in Figure 8 and $N_{T}=16$, the indexing symbols may be 


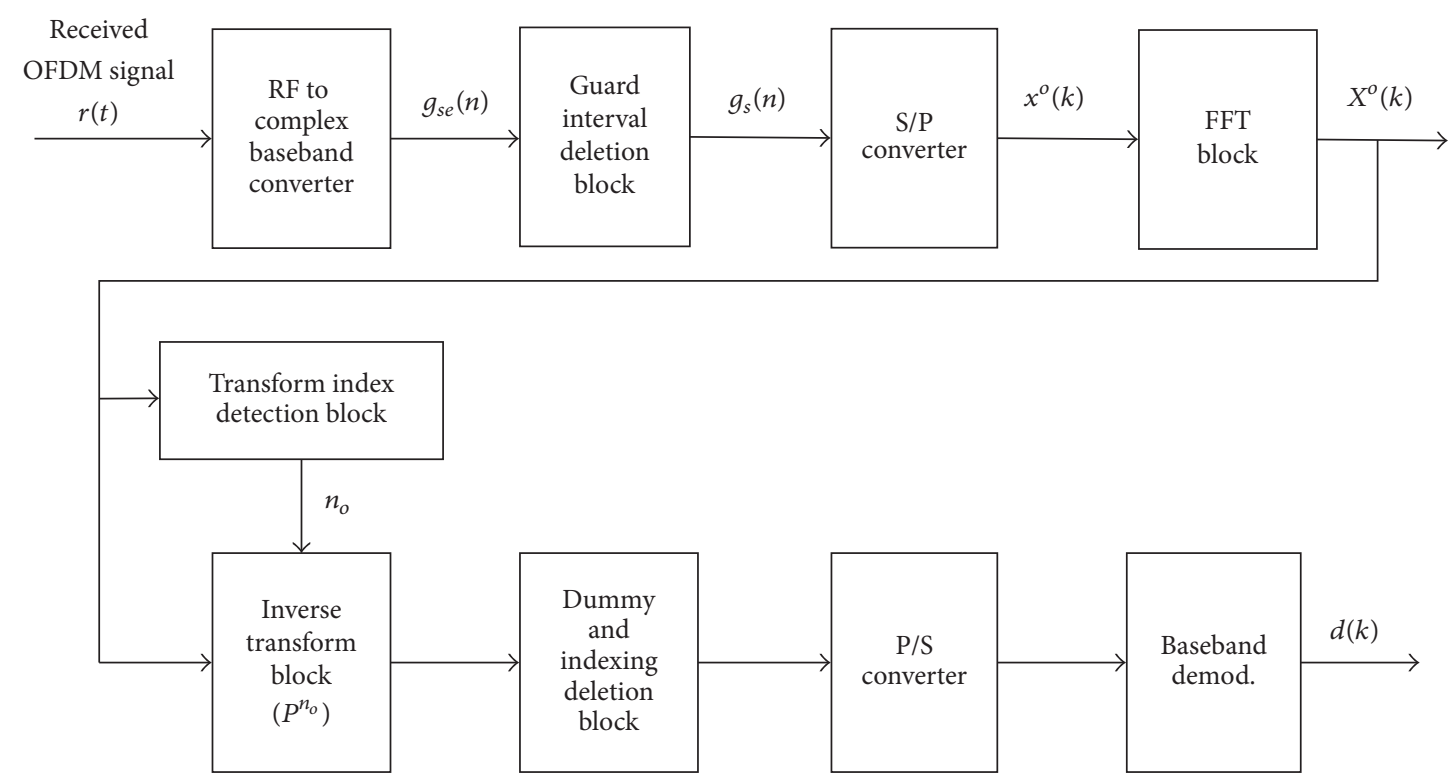

FIGURE 6: Multitransform-DSI OFDM system receiver block diagram.

selected as shown by the shaded circles in Figure 8 resulting in a minimum distance among the indexing symbols equal to $2 d$ compared to the minimum Euclidean distance equal to $d$ among the symbols in the complete signal constellation diagram, thereby minimizing the probability of detection error in the transform index $n$ in the OFDM receiver. In some cases, more than one indexing symbol may be used for further reduction of the probability of detection error. For example, using two symbols for indexing, the transform index $n$ may be encoded by a code word comprised of a pair of symbols selected from the set of symbols with indices $\{0,7,56,63\}$ in Figure 8, resulting in a minimum Euclidean distance among the code words equal to $7 d \sqrt{2} \cong 10 d$ making the probability of detection error extremely small.

\subsection{Demodulation of the Multitransform-DSI Signal. Figure 6} shows the block diagram of the receiver for the multitransform-DSI signal. The OFDM RF signal $v(t)$ received in the presence of noise $\xi(t)$ is down-converted to complex baseband and possibly filtered by a band limiting filter such as the square root raised cosine filter providing the complex baseband signal $g_{s e}(n)$ to the guard interval deletion unit that removes the guard interval from the complex baseband signal. The resulting complex baseband signal $g_{s}(n)$ is inputted to the serial to parallel converter that provides the modified signal vector at the output and given by (18) for some specific value of $n=n_{0}$ selected at the transmitter. The modified signal vector $x^{o}(k)=x^{n_{0}(k)}$ is inputted to the FFT block providing the modified modulation symbol vector $X^{n_{0}(k)}$ at the output.

The modified modulation symbol vector $X^{n_{0}(k)}$ is inputted to the transform index detection unit for detecting the transform index $n_{0}$ used in the transmitter from the vector $X^{n_{0}(k)}$. In the transform index detection unit, the vector $X^{n_{0}(k)}$ is premultiplied by the vectors $\psi^{n H}=\left[\begin{array}{llll}1 & 0 & 0 & \cdots\end{array}\right]\left(P^{n}\right)^{-1}=$ $P_{1}^{n H}$ with $P_{1}^{n H}$ denoting the conjugate transpose of the first column of the matrix $P^{n}$. With the vector $\psi^{n H}$ the metrics $r_{n}(k), n=1,2, \ldots, N_{T}$, are evaluated as in

$$
\begin{array}{r}
r_{n}(k)=\left|\psi^{n H} X^{n_{0}}(k)-s_{i}^{n}\right|^{2}=\left|P_{1}^{n H} X^{n_{0}}(k)-s_{i}^{n}\right|^{2} ; \\
n=1,2, \ldots, N_{T} .
\end{array}
$$

In (19) $s_{i}^{n}$ is the indexing symbol for the $n$th transformation matrix $P^{n}$. From (17) to (19) it follows that ignoring the receiver noise and the self-noise $i_{s}$ the index $r_{n 0}$ corresponding to the transform $P^{n 0}$ selected at the transmitter is 0 with

$$
r_{n}(k)=\left|P_{1}^{n H} P_{1}^{n_{0}} s_{i}^{n_{0}}+i_{s}-s_{i}^{n}\right|^{2} ; \quad n \neq n_{0} ; r_{n 0}=0
$$

In (20) $i_{s}$ denotes the self-noise due to the information and dummy symbols. Minimizing the metric $r_{n}$ over $n$ results in correct detection of $n_{0}$ with some small probability of error depending upon the transforms $P^{n}$ for $n=1$ through $N_{T}$ and the indexing symbols $s_{i}^{n}$. As shown in Figure 6, the transform index detection block detects the index of the transform used in the OFDM transmitter and provides the index $n_{0}$ to the inverse transform block that multiplies the OFDM modified symbol vector $X^{n_{0}(k)}$ by $\left(P^{n_{0}}\right)^{-1}=P^{n_{0} H}$. Dropping the components of $X^{n_{0}(k)}$ corresponding to the dummy symbols and the indexing symbol results in a subvector of length $N_{I}=$ $\left(N-N_{D}-1\right)$ of the information symbol vector $\bar{X}^{I}(k)$. The resulting vector is inputted to the parallel to serial converter for providing the sequence of baseband symbols $s(k)$ at the output. 
The self-noise term $i_{s}$ in (20) may be eliminated by modifying the multitransform-DSI implementation in that the $N \times N$ transform matrices $P^{n}$ are replaced by the following partitioned matrices:

$$
P^{n}=\left[\begin{array}{cc}
1 & \overline{0} \\
\overline{0}^{T} & \bar{P}^{n}
\end{array}\right] .
$$

In (21) $\overline{0}$ denotes a row vector of zeros of length $(N-1)$ and $\bar{P}^{n}$ is the $(N-1) \times(N-1)$ transform matrix obtained by deleting the first row and forts column of $P^{n}$. The vector $q^{n}$ in Figure 5 is replaced by the vector $q^{n}=P_{1}^{F} s_{i}^{n}$ where $P_{1}^{F}$ denotes the first column of the IFFT transform matrix $P^{F}$. Equivalently the indexing symbol $s_{i}^{n}$ is added to the first component of the modified information symbol vector $X^{I, n}(k)$. In the modified approach, the indexing symbol is not a part of the transform operation. In the demodulation of the multitransform OFDM signal, Figure 6 is modified accordingly. Thus in the transform index detection unit, the first element $X_{1}^{n_{0}}$ of the vector $X^{n 0}(k)$ that is equal to the indexing symbol $s_{i}^{n_{0}}$ plus receiver noise $\xi(k)$ is used to detect the transform index by the minimization

$$
\min _{n}\left|X_{1}^{n_{0}}-s_{i}^{n}\right|^{2}=\min _{n}\left|s_{i}^{n_{0}}+\xi(k)-s_{i}^{n}\right|^{2}
$$

Except for the change in the operation of the transform index detection unit, the block diagram of the modified multitransform OFDM system is same as that in Figure 6. Partition similar to that in (21) may be used for transmission of any pilot symbols.

The following section presents the simulation results on the performance of the multitransform PAPR reduction OFDM system and compares it with some of the existing methods for the reduction of the PAPR.

\section{Simulation Results}

This section presents simulation results on the performance of the multitransform techniques for the PAPR (peak to average power ratio) reduction with a performance comparison with the existing precoding and dummy sequence insertion (DSI) based techniques that may be the ones most promising in terms of the various criteria including minimal reduction in bandwidth efficiency and distortion less transformation. The results for the existing methods are similar to those in the various references of this paper. The complementary cumulative probability distribution function (CCDF) of the PAPR is obtained by simulation runs of $10^{4}$ OFDM symbols for all possible FFT length $N$. Thus the number of QAM symbols simulated in each run is equal to $N \times 10^{4}$. The simulations are performed with MQAM modulation with the number of points $M$ in the signal constellation selected equal to 16,64 , and 256. In case of the dummy sequence insertion scheme, the number of dummy symbols is limited to 1 in all of the simulation results as an increase in the length of the dummy sequence provided only marginal improvement at the cost

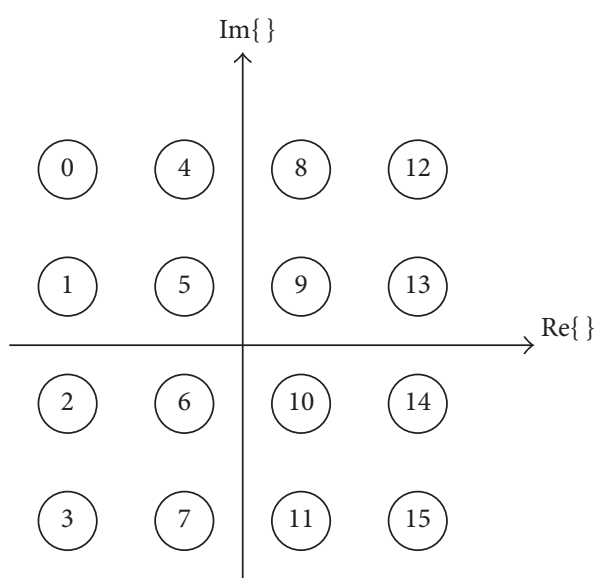

FigUre 7: Signal constellation diagram for 16 QAM signal.

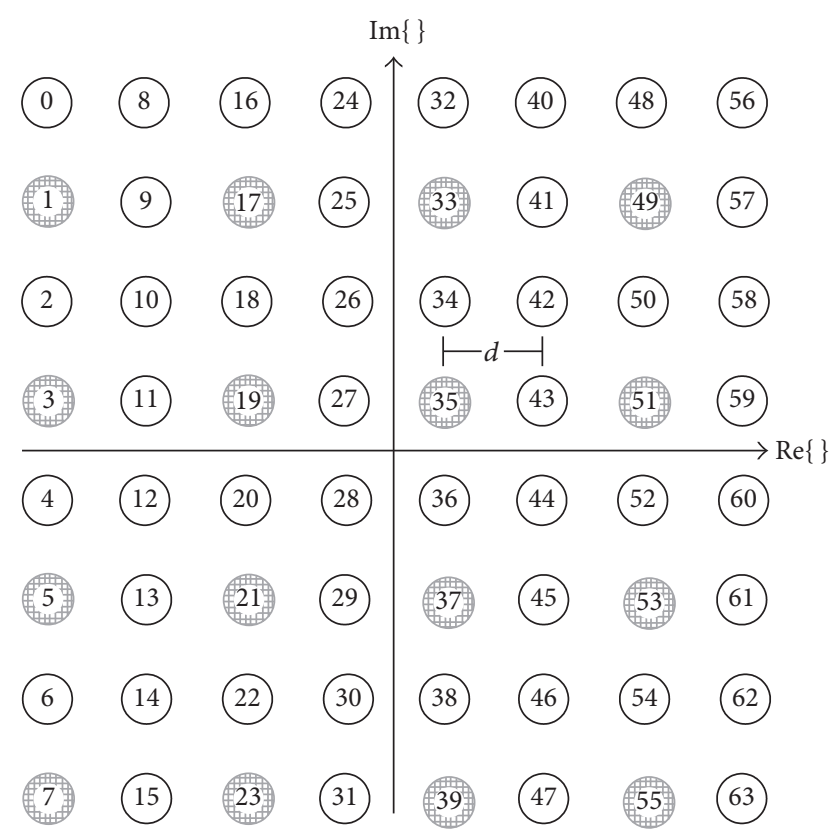

FIGURE 8: Signal constellation diagram for 64 QAM signal.

of significant increase in computational complexity. In the constellation diagrams, the symbols are indexed using the Grey coding scheme. Figures 7 and 8 show the constellation diagram for the case of $M$ equal to 16 and 64, respectively.

Figure 9 plots the result for the case of the $N=64$ subcarriers and 16 QAM modulation with and without the dummy symbol insertion wherein the dummy symbol is selected to be any of the 16 possible points in the constellation diagram. Different possible values of the dummy symbol are selected until an improvement in the PAPR exceeds the specified threshold value of $0.4 \mathrm{~dB}$. As may be inferred from Figure 9, an improvement of about $0.4 \mathrm{~dB}$ is achieved with the insertion of the dummy symbol. Figure 10 shows the corresponding result when all 16 possible symbol values are tried in an exhaustive manner and the one providing the most improvement in the PAPR is selected. As may be inferred 


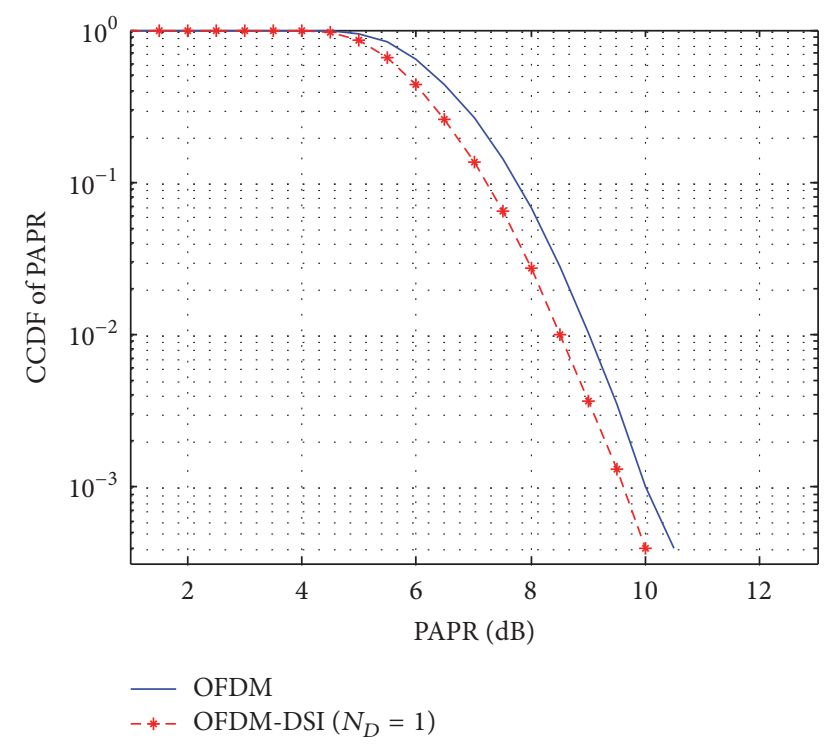

FIGURE 9: CCDF of OFDM-DSI system for 16 QAM (0.4 dB threshold).

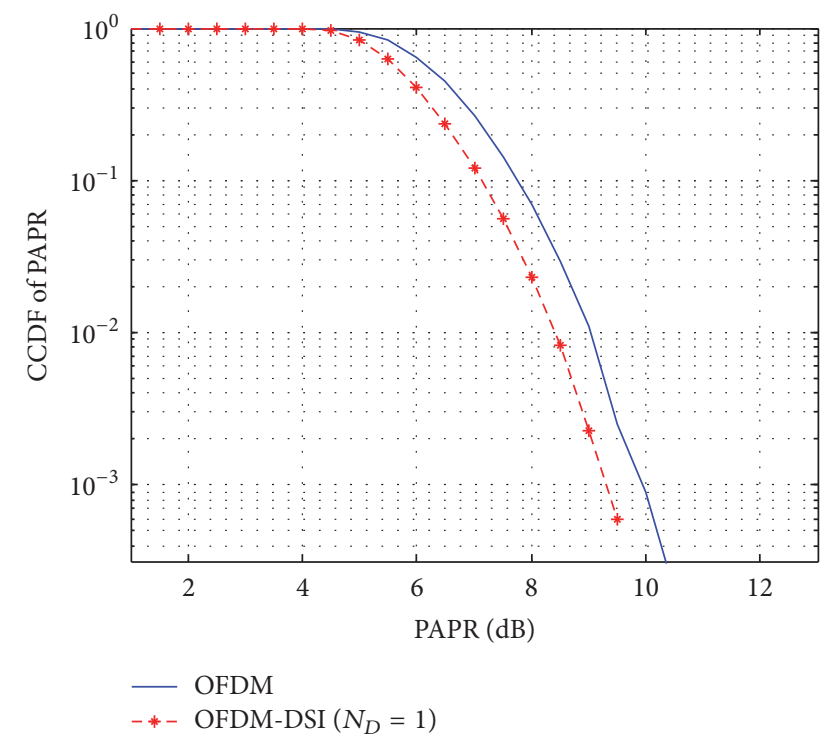

FIGURE 10: CCDF of OFDM-DSI system for 16 QAM (exhaustive search).

from Figure 10, the improvement in the PAPR is about $0.6 \mathrm{~dB}$ at the CCDF value of $10^{-3}$ compared to the value of $0.4 \mathrm{~dB}$ in Figure 9. Figure 11 shows the histogram of the index of the dummy symbol selected in the simulation result of Figure 10. It is interesting to observe that 4 of the possible values of the dummy sequence are selected with much higher probability compared to the other 12 values. This result may provide further insight into the selection of the dummy sequence.

Similar PAPR improvement is obtained for the case of 64 QAM modulation format wherein a reduction of about $0.7 \mathrm{~dB}$ is achieved at the CCDF value of $10^{-3}$ when the dummy symbol is varied over all possible 64 values. The detailed

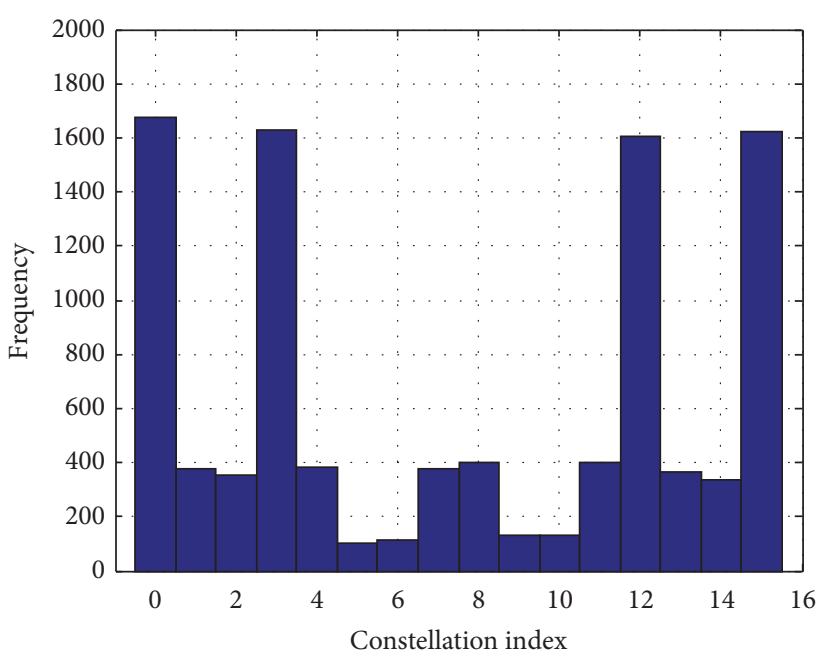

FIGURE 11: Histogram of the dummy symbol index selected in the simulation example of Figure 10.

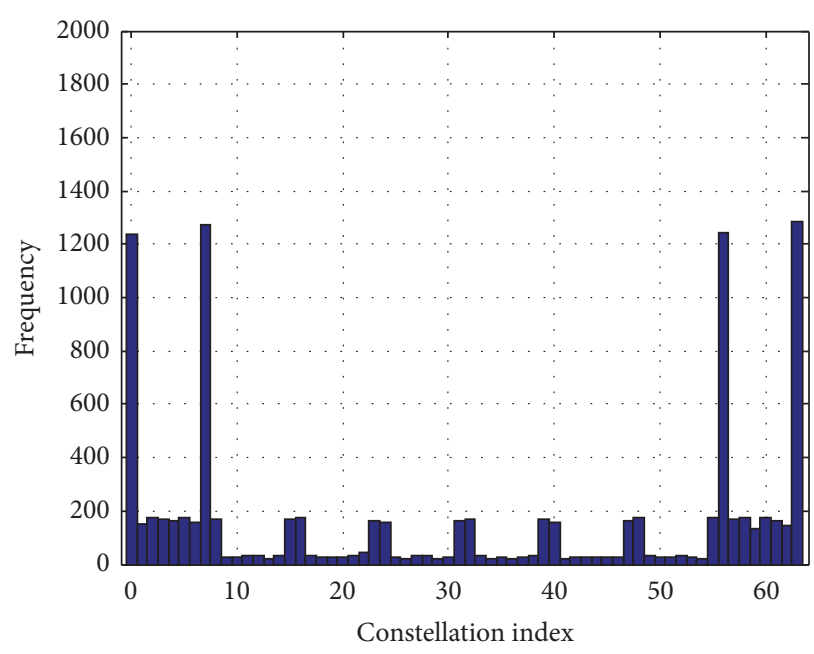

FIGURE 12: Histogram of the dummy symbol index selected for the case of 64 QAM modulation.

graph for the CCDF for this case is not included for the sake of brevity; however, the histogram of the selected symbol is shown in Figure 12. Examination of Figure 12 again shows the interesting result that the histogram has sharp peaks at four of the 64 possible values in the constellation diagram. Interestingly this result is very similar to that of Figure 11. Thus it is possible to reduce the number of trials for the dummy symbol to 4 without any significant degradation in performance. This in fact is done in some of the simulations presented latter in the paper.

Another technique used for the reduction of the peak to average power reduction consists of precoding the modulation symbol vector by a transform matrix. The simulation results are presented next to evaluate the PAPR performance with the precoding techniques. The simulation results when both the precoding and dummy sequence are used simultaneously are presented as well. 
TABLE 1: PAPR Improvement in $\mathrm{dB}$ at CCDF of $10^{-3}$ for the precoding, DSI, and the hybrid precoding-DSI methods (PAPR $=10 \mathrm{~dB}$ for standard OFDM).

\begin{tabular}{|c|c|c|c|c|}
\hline & \multicolumn{2}{|c|}{16 QAM } & \multicolumn{2}{|c|}{64 QAM } \\
\hline & Number of recursions & Improvement in PAPR $(\mathrm{dB})$ & Number of recursions & $\begin{array}{l}\text { Improvement in } \\
\text { PAPR }(\mathrm{dB})\end{array}$ \\
\hline OFDM & 1 & - & 1 & - \\
\hline WHT & 1 & 1.07 & 1 & 0.94 \\
\hline DCT & 1 & 1.84 & 1 & 1.80 \\
\hline DHT & 1 & 3.70 & 1 & 3.04 \\
\hline $\operatorname{DSI}\left(V_{T}=0.4\right)$ & 8.69 & 0.39 & 14.41 & 0.48 \\
\hline DSI (All) & 16 & 0.64 & 64 & 0.78 \\
\hline WHT-DSI $\left(V_{T}=0.1\right)$ & 8.41 & 1.11 & 44.49 & 1.26 \\
\hline WHT-DSI $\left(V_{T}=0.2\right)$ & 11.03 & 1.17 & $\left(V_{T}=0.3\right)$ & $\left(V_{T}=0.3\right)$ \\
\hline WHT-DSI (All) & 16 & 1.30 & 64 & 1.41 \\
\hline DCT-DSI $\left(V_{T}=0.1\right)$ & 8.09 & 1.96 & 16.64 & 1.77 \\
\hline DCT-DSI $\left(V_{T}=0.2\right)$ & 13.36 & 2.09 & - & - \\
\hline DCT-DSI (All) & 16 & 2.13 & 64 & 1.90 \\
\hline DHT-DSI $\left(V_{T}=0.06\right)$ & 7.23 & 3.78 & 16.58 & 3.16 \\
\hline DHT-DSI $\left(V_{T}=0.08\right)$ & 10.21 & 3.79 & - & - \\
\hline DHT-DSI (All) & 16 & 3.81 & 64 & 3.18 \\
\hline
\end{tabular}

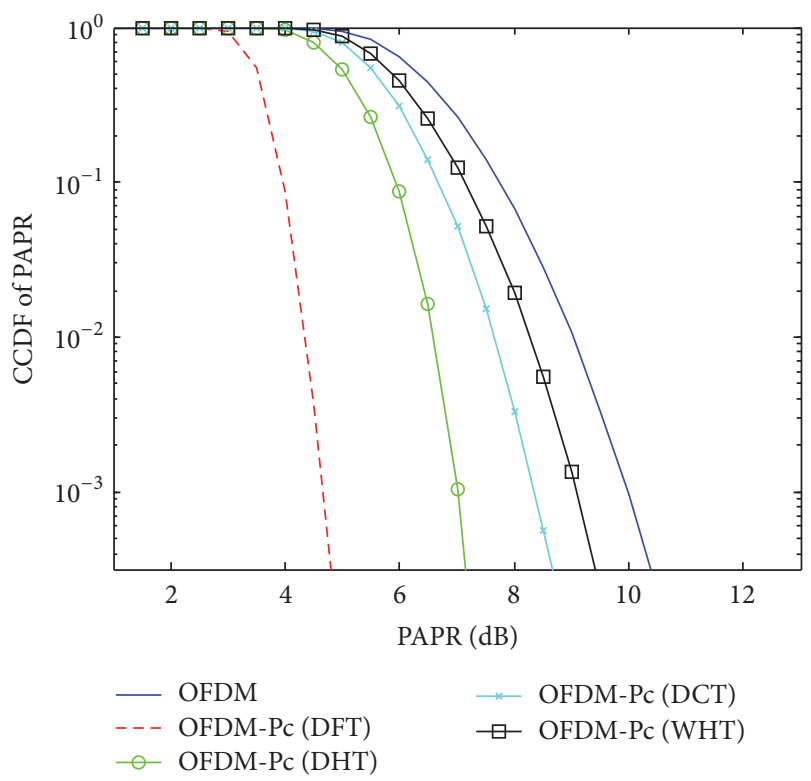

Figure 13: Comparison of the CCDF of the peak to average power ratio with different precoding matrices.

Figure 13 plots the CCDF of the PAPR for the 16 QAM modulation and 64 subcarriers case when the precoding technique is used. Three different precoding matrices of the published literature, namely, the discrete Hartley transform (DHT), discrete cosine transform (DCT), and the WalshHadamard transform (WHT) matrices, are considered for the simulations. In Figure 13 and subsequent figures, the abbreviation Pc stands for precoding. Thus OFDM-Pc(DHT) denotes the case of the OFDM system with precoding based on the discrete Hartley transform. The figure also includes the case of using the DFT transform for precoding which reduces the OFDM system to a single carrier system.

As may be inferred from Figure 13 the discrete Hartley transform provides the best performance among the three transforms with a reduction of $3.7 \mathrm{~dB}$ in PAPR at CCDF of $10^{-3}$ with the DCT and WHT providing a reduction of $1.84 \mathrm{~dB}$ and $1.07 \mathrm{~dB}$, respectively. The results obtained for the precoding method, the DSI method and the hybrid precoding plus DSI (Pc-DSI) method are summarized in Table 1 in terms of the reduction in the PAPR at the CCDF value of $10^{-3}$ for these methods. The results in Table 1 include both methods of selecting the optimum dummy symbol. In the first method the search is continued until the PAPR improvement exceeds certain specified threshold $V_{T}$ selected to be $0.1 \mathrm{~dB}$ and $0.2 \mathrm{~dB}$ in the table. In the second method all possible $M$ symbols are tried in selecting the optimum dummy symbol. The results for the two cases of $M=16$ and $M=64$ are included in the table. The table also includes the average number of recursions for the case of DSI and the hybrid Pc-DSI methods. In terms of a direct implementation, one recursion for the case of precoding techniques involves 1 matrix vector multiplication and $1 \mathrm{~N}$-point IFFT operation. For the case of DSI, it involves one IFFT operation and for the hybrid Pc-DSI method, one recursion involves 1 matrix vector multiplication and $1 \mathrm{~N}$-point IFFT operation. The computational requirements may be reduced by appropriate reorganization of the computations as shown in the previous section of the paper.

Examination of Table 1 shows that the precoding method with discrete Hartley transform (DHT) provides the best performance among the precoding techniques with a PAPR improvement of $3.7 \mathrm{~dB}$ and $3.0 \mathrm{~dB}$, respectively, for the case 
TABLE 2: Indices of the transforms $\left(N_{T}=16\right)$.

\begin{tabular}{|c|c|}
\hline Index & Transform \\
\hline 1 & I \\
\hline 2 & DHT \\
\hline 3 & DCT \\
\hline 4 & WHT \\
\hline 5 & $\mathrm{DHT} * \mathrm{DCT}$ \\
\hline 6 & $\mathrm{DHT} * \mathrm{WHT}$ \\
\hline 7 & $\mathrm{DCT} * \mathrm{DHT}$ \\
\hline 8 & $\mathrm{DCT} * \mathrm{WHT}$ \\
\hline 9 & $\mathrm{WHT} * \mathrm{DHT}$ \\
\hline 10 & $\mathrm{WHT} * \mathrm{DCT}$ \\
\hline 11 & $\mathrm{DHT} * \mathrm{DCT} * \mathrm{WHT}$ \\
\hline 12 & $\mathrm{DHT} * \mathrm{WHT} * \mathrm{DCT}$ \\
\hline 13 & $\mathrm{DCT} * \mathrm{DHT} * \mathrm{WHT}$ \\
\hline 14 & $\mathrm{DCT} * \mathrm{WHT} * \mathrm{DHT}$ \\
\hline 15 & $\mathrm{WHT} * \mathrm{DHT} * \mathrm{DCT}$ \\
\hline 16 & $\mathrm{WHT} * \mathrm{DCT} * \mathrm{DHT}$ \\
\hline
\end{tabular}

of $M$ equal to 16 and 64, respectively. Including the DSI with the DHT precoding improves the PAPR by an additional $0.1-$ $0.2 \mathrm{~dB}$. Increasing the number of dummy symbols may result in some marginal increase in performance but at the cost of higher computational complexity.

Figure 14 shows the CCDF of the PAPR obtained with the multiple transform technique wherein one of the $N_{T}$ possible transform matrices including the case of no transform is selected to optimize the PAPR in each OFDM frame shown as OFDM-OP (OFDM with optimum transform) in the figure and compares it with that obtained with the fixed transform methods. Table 2 lists the various transforms used in the simulations presented in Figure 14.

In Figure 14 the number of subcarriers is 64 and 64 QAM modulation is considered. As may be inferred from Figure 14 the optimum transform provides an improvement of about $1.3 \mathrm{~dB}$ over the discrete Hartley transform that has the best performance among all of the fixed transform methods. Figure 15 plots the corresponding result for the case of 256 QAM modulation showing an improvement of about $1.4 \mathrm{~dB}$ compared to the best of the previous schemes. This is remarkable in that the PAPR obtained with the use of the optimum transform method is only about $0.9 \mathrm{~dB}$ worse compared to the single carrier system with a high order modulation.

Figure 16 shows the histogram of the number of times a transform is optimum and is selected for the PAPR reduction. As may be inferred from the figure, the DHT transform is optimum most often followed by DCT and WHT transforms with the remaining cases occurring with about uniform probability. This may not come as a surprise as among the fixed transform methods the DHT provides the best performance. However, it is not the best among all the cases and that is where the performance improvement comes from.

Figure 17 shows the simulation result for the optimum transform method along with the use of one dummy symbol

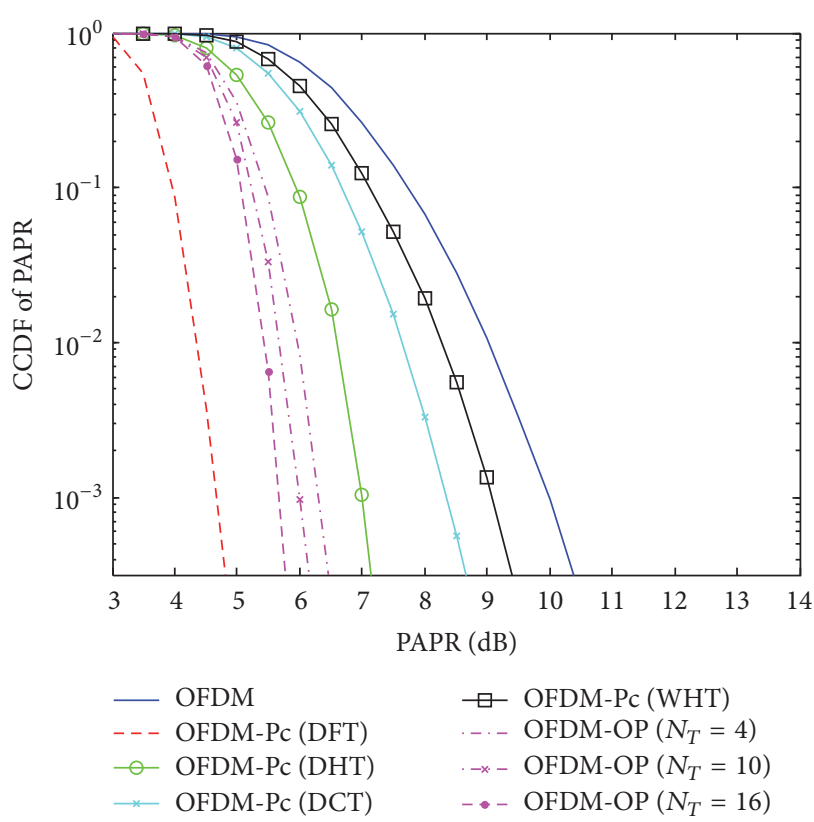

FIGURE 14: Comparison of the CCDF of the PAPR obtained with various transform methods (64 QAM).

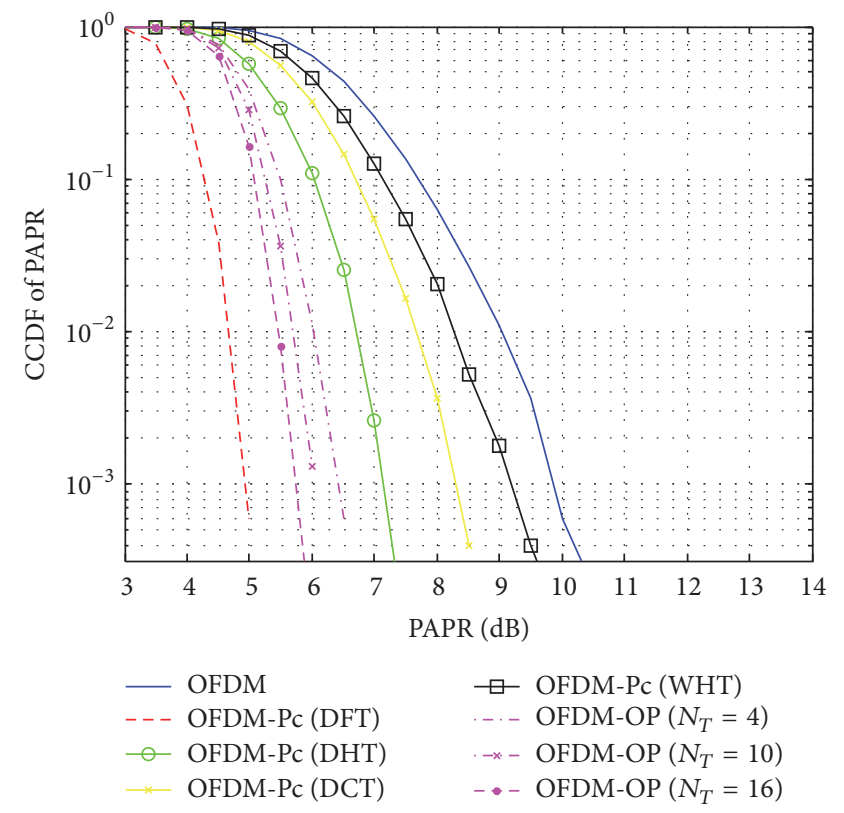

FIGURE 15: Comparison of the CCDF of the PAPR obtained with various transform methods (256 QAM).

along with the result obtained with the fixed transform methods for reference. As an interesting case Figure 16 also includes the case wherein the transform selection is limited to only four cases, namely, DFT that is equivalent to single carrier case, WHT, DCT, and the DHT. The case of hybrid DHT-DSI has already been considered in Table 1 showing that the marginal improvement due to DSI when used with the DHT is limited to about $0.1 \mathrm{~dB}$ and is not included in Figure 17 for clarity. As may be inferred from Figure 17, the proposed 


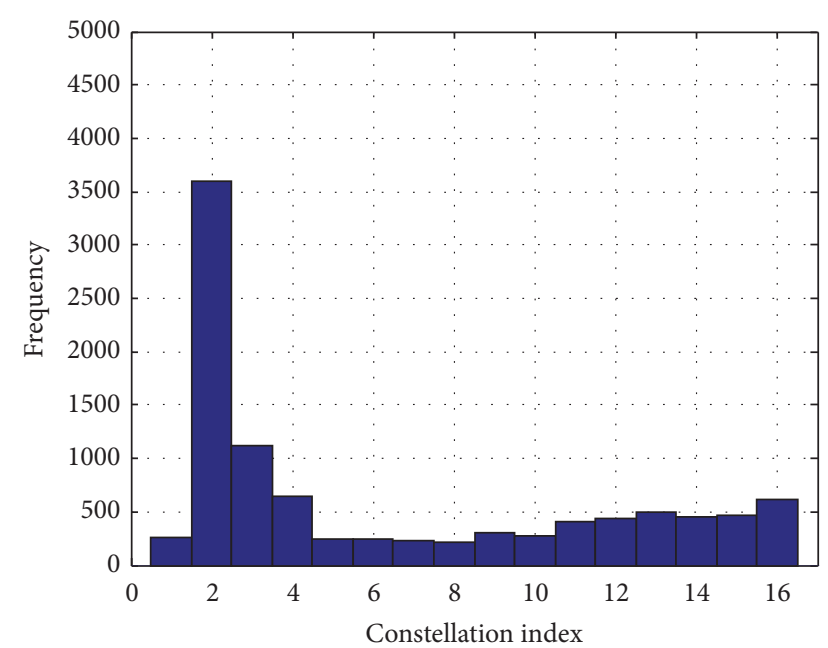

FIGURE 16: Histogram of the frequency of selection of the various transforms.

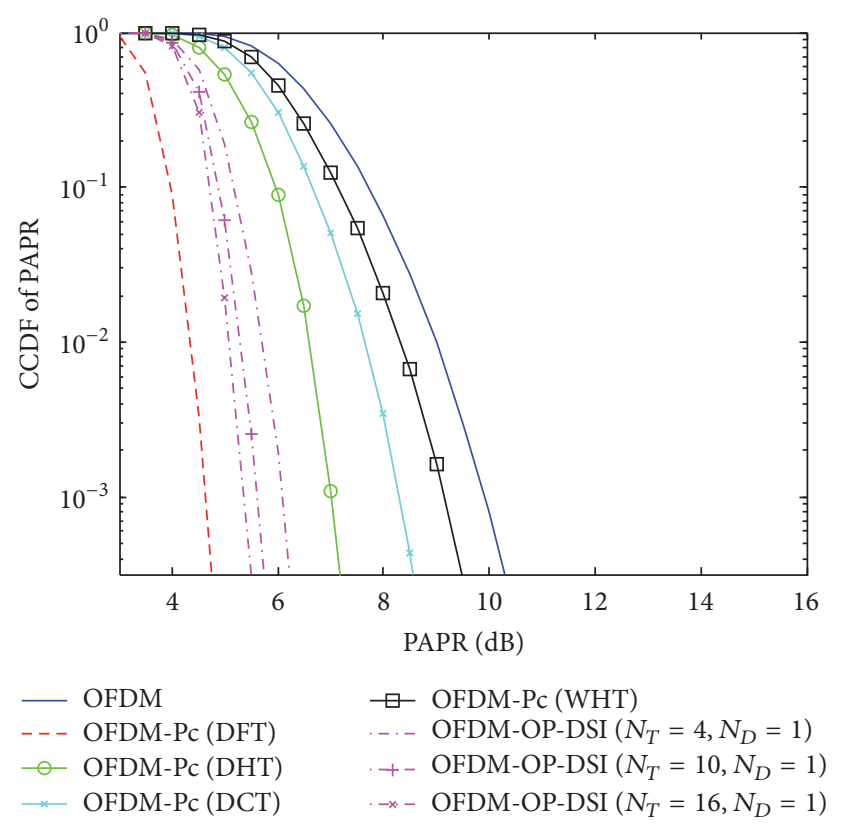

FIGURE 17: PAPR performance of the OFDM-OP-DSI method in comparison with the other methods.

OFDM-OP-DSI method provides an improvement of about $1.75 \mathrm{~dB}$ in PAPR over the DHT method, and what is perhaps even more remarkable is the fact that it is only about $0.45 \mathrm{~dB}$ worse compared to case of a single carrier. It is interesting to note that when the transform selection is limited to only 4 cases mentioned earlier, the improvement is only about one half of that with the full selection of the 16 transforms. Thus it is the combination of the wide selection of the transforms along with the dummy symbol that provides the maximum reduction in the PAPR. The histogram of the frequency of selection of the various transforms is very similar to that in Figure 16 and is not presented here.

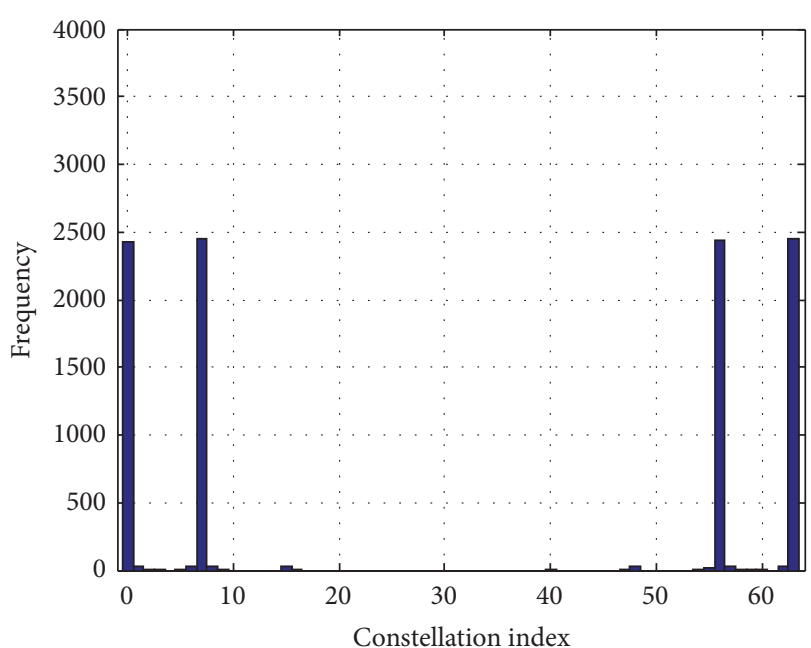

FIGURE 18: Histogram of the symbol frequency for the optimum transform method.

The results in Figure 17 are obtained when the dummy symbol is selected optimally by an exhaustive search. Therefore, it is of interest to find if there is any specific pattern in the selection of the dummy symbol. Figure 18 shows the histogram of the dummy symbol selection frequency for the case of the OFDM-OP-DSI method. As may be inferred from the figure, the histogram shows four distinct peaks that correspond to the boundary symbols $0,7,56$, and 63 shown in the signal constellation diagram of Figure 8.

When the selection of the dummy symbol is restricted to one of the four symbols $\{0,7,56,63\}$ with the highest frequencies in the histogram of Figure 18, the performance in terms of PAPR is very close to that obtained in Figure 17. Figure 19 plots the PAPR result for the multitransform-DSI case on an expanded scale when the number of transforms $N_{T}$ is equal to 16 and wherein $N_{W}$ denotes the number of symbols in the signal constellation over which the dummy symbol is optimized. As may be inferred for the figure, for a CCDF value of $10^{-3}$, the PAPR is equal to about $5.25 \mathrm{~dB}$ with $N_{W}=64$. For the case wherein the selection of the dummy symbol is restricted to $N_{W}=4$ symbols, the corresponding value of PAPR is about $0.1 \mathrm{~dB}$ higher. Thus with only a relatively very small increase in the PAPR, the computational complexity can be reduced by an order of magnitude. In another alternative method, the dummy symbol is selected on the basis of a threshold on the PAPR. In this method, for the selected symbol, the PAPR is evaluated and compared with the PAPR evaluated for the standard OFDM for the same OFDM frame. If the PAPR for the multitransform-DSI method exceeds the threshold, the search is discontinued; otherwise another dummy symbol is tried. The threshold is equal to PAPR value predicted for the multitransform-DSI method at the CCDF value read from the CCDF versus PAPR graph for the OFDM method at the computed PAPR value for the OFDM method minus the specified value of $V_{T}$. In the threshold method, first the symbols in the set $\{0,7,56,63\}$ are selected followed by selection of other symbols in the signal constellation. Figure 19 shows the PAPR performance for the 
TABLE 3: PAPR of the OFDM-OP-DSI method for the reduction in PAPR (64 QAM, 64 FFT).

\begin{tabular}{|c|c|c|c|}
\hline Parameters of the PAPR reduction method & PAPR (dB) & Reduction in PAPR $(\mathrm{dB})$ & $\triangle \mathrm{PAPR}$ \\
\hline Standard OFDM & 10 & - & \\
\hline$N_{T}=3, N_{W}=64$ & 6.22 & 3.78 & 1.46 \\
\hline$N_{T}=12, N_{W}=64$ & 5.54 & 4.46 & 0.78 \\
\hline$N_{T}=16, N_{W}=64$ & 5.25 & 4.75 & 0.49 \\
\hline$N_{T}=3, N_{W}=4$ & 6.25 & 3.75 & 1.49 \\
\hline$N_{T}=12, N_{W}=4$ & 5.82 & 4.18 & 1.06 \\
\hline$N_{T}=16, N_{W}=4$ & 5.30 & 4.70 & 0.55 \\
\hline Single carrier system & 4.76 & - & 0 \\
\hline
\end{tabular}

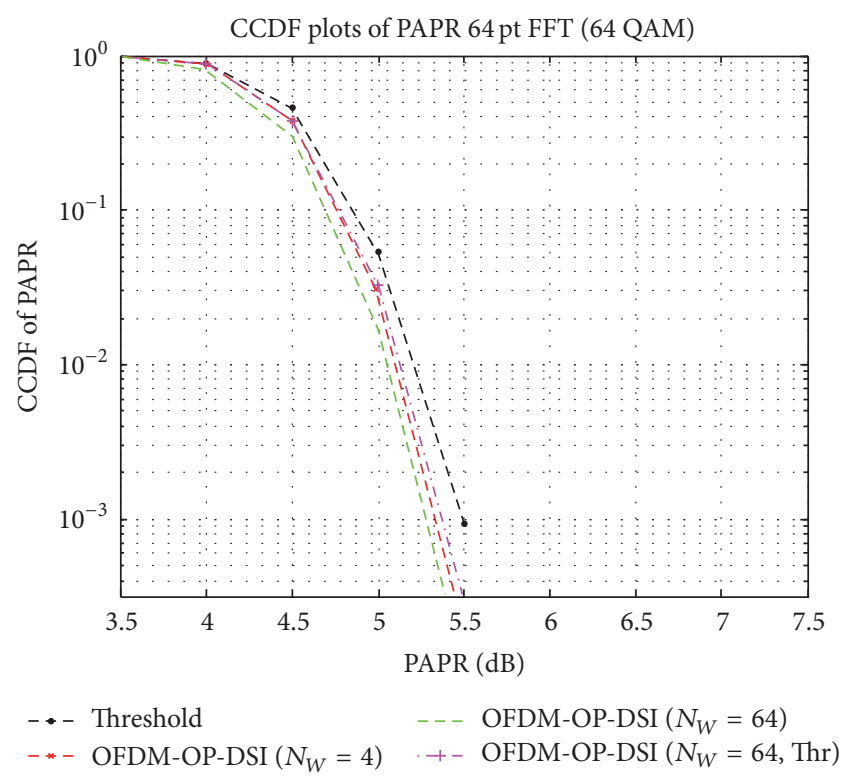

FIGURE 19: PAPR performance of the OFDM-OP-DSI method $\left(N_{T}\right.$ $=16$ ).

multitransform-DSI threshold method for both $N_{W}=64$ and $N_{W}=4$. As may be inferred from Figure 19, the performance for the threshold case with $N_{W}=64$ is about the same as for the case of exhaustive search with $N_{W}=4$.

Table 3 summarizes the PAPR at a CCDF value of $10^{-3}$ for the OFDM-OP-DSI method wherein $N_{W}$ denotes the number of possible symbols over which the dummy symbol selection is optimized and $N_{T}$ is number of possible transforms. The table includes the case of single carrier transmission for comparison and also lists the $\triangle$ PAPR defined as the difference between the PAPR achieved with the OFDMOP-DSI method and that of the single carrier transmission at the CCDF value of $10^{-3}$.

As may be inferred from Table 3 the OFDM-OP-DSI system with $N_{T}=12$ provides an improvement of $4.46 \mathrm{~dB}$ in PAPR over the OFDM with the resulting PAPR only $0.78 \mathrm{~dB}$ higher than for the single carrier transmission. With $N_{T}=$ 16 the improvement is $4.75 \mathrm{~dB}$ in PAPR over the OFDM with the resulting PAPR only $0.49 \mathrm{~dB}$ higher than for the single carrier transmission thus almost entirely eliminating the PAPR limitation of the OFDM system. It may be possible

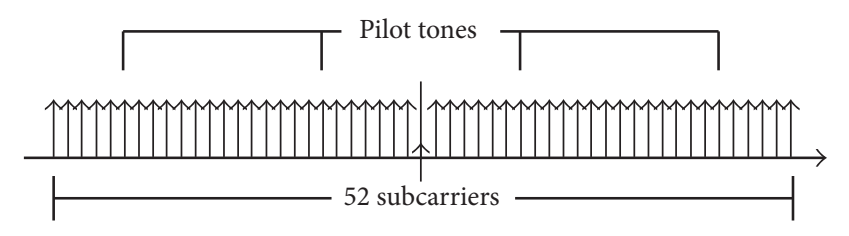

FIGURE 20: Subcarrier layout for IEEE OFDM standard (64 subcarriers).

to reduce this gap of $0.49 \mathrm{~dB}$ further by increasing the number of dummy symbols to more than 1 and/or by increasing the number of total transforms beyond 16 considered in the paper at the expense of some additional computations. On the other hand, the computational complexity can be significantly reduced by restricting the number of symbols $N_{W}$ from which the dummy symbol is selected at the cost of some increase in the PAPR. As shown in the table, with $N_{W}=4$, and $N_{T}=16$, the PAPR reduction is $4.70 \mathrm{~dB}$ compared to $4.75 \mathrm{~dB}$ with $N_{W}=64$. For the case of $N_{T}=12$, and $N_{W}=4$, the PAPR reduction is $4.18 \mathrm{~dB}$ compared to $4.46 \mathrm{~dB}$ with $N_{W}=64$, thus resulting in a relatively small degradation in PAPR due to the reduction in $N_{W}$ from 64 to 4. However, the reduction in $N_{W}$ from 64 to 4 results in an order of magnitude reduction in the computational requirements. Thus the OFDM-OP-DSI method provides a PAPR that is only about $0.5 \mathrm{~dB}$ worse than for the single carrier system with only moderate computational requirements. This gap of about $0.5 \mathrm{~dB}$ may be further reduced by selecting the number of transforms $N_{T}$ to be higher than 16 .

5.1. Results for the Case of IEEE 802.11 Standard. The simulation results presented thus far are for the ideal case when all of the $N$ subcarriers are used for transmission including those allocated to the dummy symbols and the transform indices. However, in various OFDM standards some of the subcarriers near the band edge are not used for transmission in order to minimize the degradation due to the tapering in the frequency response of the band limiting transmission filter. For example, as shown in Figure 20, in the IEEE 802.11 standard with $N=64$, only 52 subcarriers are used for transmission with 12 subcarriers not used for transmission. Out of the 52 subcarriers used for transmission, 4 of these are for pilot tones with 48 used for data transmission. 


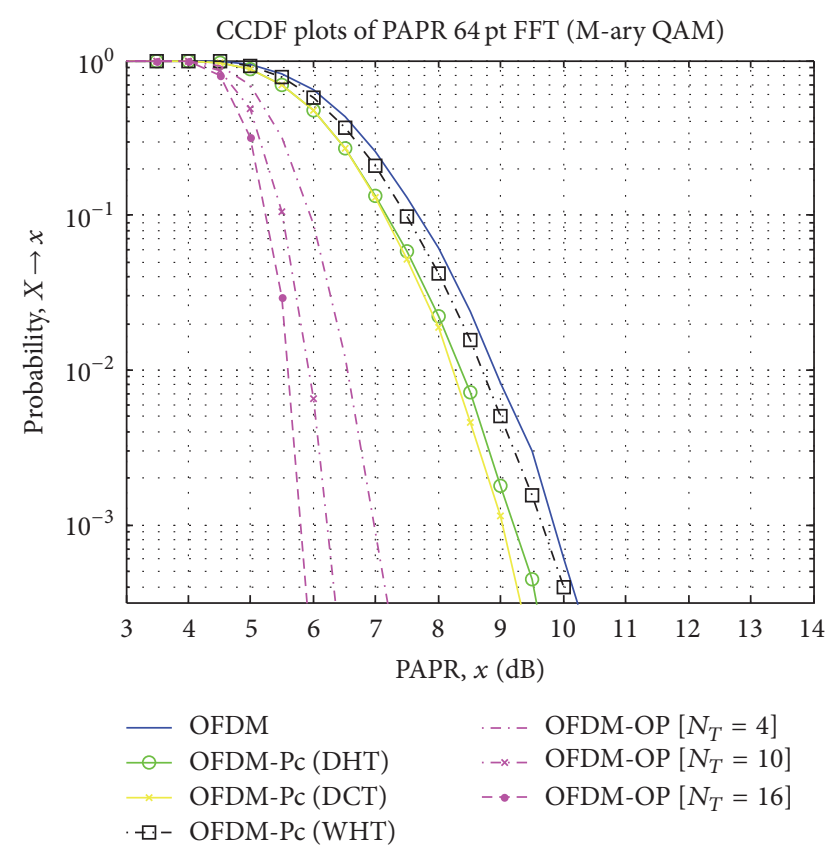

FIgURE 21: Comparison of the CCDF of the PAPR obtained with various transform methods (IEEE standard, 64 QAM).

Figure 21 plots the result for the complementary cumulative distribution function (CCDF) of the paper for various transform techniques including the multitransform method of the paper with the use of 48-point orthonormal transforms.

Comparison of Figure 21 with Figure 14 shows that for the case of multitransform method there is no significant difference in performance between the ideal case of 64 point transform and the 48-point transform except that for the case of $N_{T}=4,48$-point transform the PAPR is about $0.4 \mathrm{~dB}$ higher compared to the case of $N_{T}=4,64$-point transform at the CCDF value of $10^{-3}$. Such a result may also be intuitively expected. However, similar comparison for the fixed transforms case shows a drastic difference in performance. For example, for the DHT transform, the PAPR value for the case of 48-point transform case is about $9.2 \mathrm{~dB}$ compared to the value of about $7.2 \mathrm{~dB}$ for the 64 point transform case showing that being with some of the subcarriers with no transmission reduces the effectiveness of the fixed DHT orthonormal transform. The result for the case of DCT and WHT transforms is not as drastic; however, the improvement in PAPR with either of these transforms is relatively small in both of the 48-point and 64point transform cases. As may be inferred from Figure 21, for the 48-point case relevant to the IEEE standard, the multitransform method provides an improvement of about $3.6 \mathrm{~dB}$ over the best fixed transform method at a CCDF value of $10^{-3}$.

Figure 22 plots the CCDF of the PAPR obtained with the composite transform and dummy symbol insertion (DSI) methods with $N_{D}=1$ for the case of IEEE standard 48point transform. Comparison of Figure 22 shows that the results obtained are very similar to that obtained for the

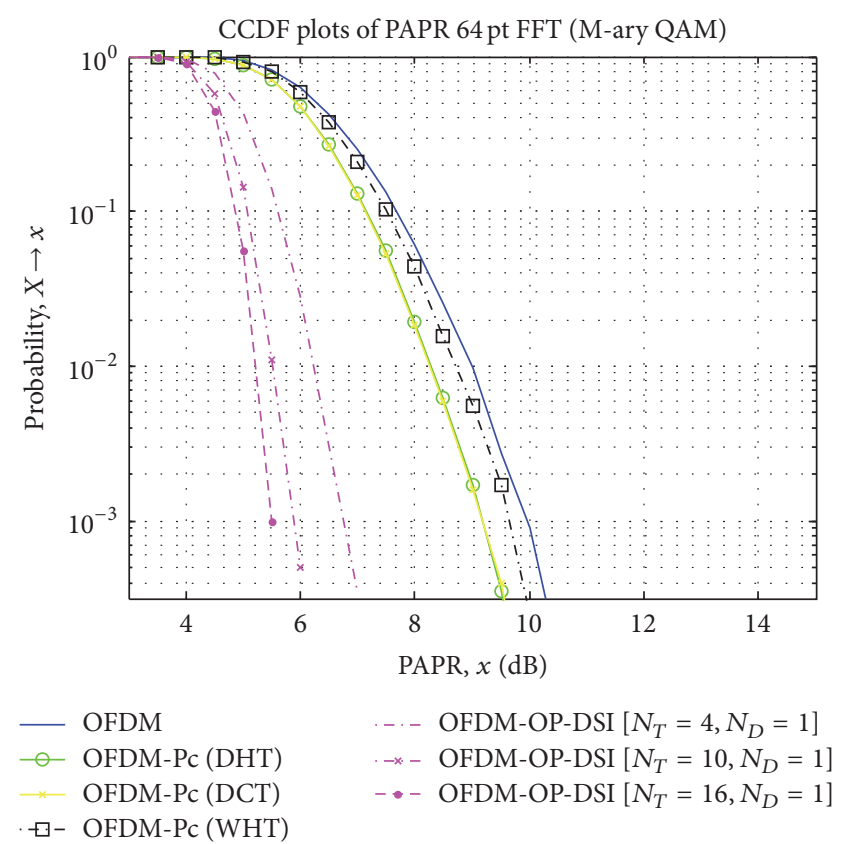

FIGURE 22: PAPR performance of the OFDM-OP-DSI method in comparison with the other methods (IEEE standard, 64 QAM).

transform methods in Figure 21. For the multitransform the DSI reduces the PAPR to about $5.4 \mathrm{~dB}$ compared to the value of about $5.9 \mathrm{~dB}$ obtained without DSI for the case of $N_{T}$ 16. However, for the case of fixed transform, there is no significant improvement in the PAPR due to the use of a single dummy symbol. Simulation results with $N_{D}=2$ show that the multitransform method with DSI provides a PAPR value of about $4.9 \mathrm{~dB}$ with $N_{T}=16$ at a CCDF value of $10^{-3}$. In comparison there is no appreciable improvement for the fixed transform methods with $N_{D}=2$. When the search for the dummy symbols is restricted to the 4 boundary symbols given by $\{0,7,56,63\}$ in the constellation diagram of Figure 8 , there is only a minor degradation of about $0.1 \mathrm{~dB}$ in the PAPR reduction.

\section{Conclusions}

The paper has presented multitransform methods and systems taught in US Patent 8,995,542, March 31, 2015, for the reduction of peak to average power ratio (PAPR) in OFDM systems. Simulation results on the performance of various PAPR reduction techniques for the OFDM systems are presented. Detailed simulation results have been presented on the comparison of the performance of various fixed precoding transform techniques and the multitransform technique with and without the dummy symbol insertion (DSI) techniques.

The fixed transform techniques include the discrete Hartley transform (DHT), Walsh-Hadamard transform (WHT), and discrete cosine transform (DCT). The transform techniques with or without DSI have a reasonable computational requirement, do not introduce any distortion, and may 
introduce relatively small decrease in the bandwidth efficiency. In fact the recently invented multitransform-DSI techniques need only 1 or 2 dummy symbols to obtain optimum performance and thus possess high bandwidth efficiency as well.

In terms of the dummy symbol insertion method, the dummy symbol selection is made by an exhaustive optimization over the constellation diagram of the modulated symbols. It is observed that in the simulated examples the optimum symbol belongs to a small subset of four symbols with a probability close to 1 . Thus in actual implementation, the selection of the dummy symbol may be confined to such a subset resulting in significant reduction in computational effort without causing any significant reduction in the PAPR performance compared to the case wherein the selection is made from the complete symbol constellation.

The simulation results have been presented for two general cases. In the first of these cases, all of the OFDM channels are included in the transform operation. In the second case applicable to the IEEE 802.11 standard, only a subset of the OFDM channels is included in the transform operation with either the remaining channels carrying zeros or the pilot symbols. The pilot symbols are not included in the transform operation for ease of subcarrier frequency synchronization although synchronization methods may be devised for the first case as well.

Simulation results show that among the fixed transform methods the DHT method provides the best performance. For example, for the case where all the channels are included in the transform operation and with 64 QAM modulation, the DHT method provides a PAPR of about $7 \mathrm{~dB}$ corresponding to a CCDF of $10^{-3}$ or with a probability of 0.999 , with the corresponding values of the PAPR for the DCT and WHT equal to about $8.7 \mathrm{~dB}$ and $9.3 \mathrm{~dB}$, respectively. For the multitransform technique with $N_{T}$ equal to 16 , the value of PAPR is about $5.8 \mathrm{~dB}$ providing an improvement of $1.2 \mathrm{~dB}$ over the best fixed transform. Similar results are obtained for other modulation schemes. Similar differences in PAPR for various fixed and multitransform methods are observed for the case of 256 QAM. The multicarrier penalty in terms of increased PAPR is reduced to only $0.8 \mathrm{~dB}$ with the use of the multitransform technique and represents a reduction of about $4.5 \mathrm{~dB}$ compared to the original OFDM without any precoding transform.

In terms of the composite transform-DSI technique, insertion of 1 or 2 dummy symbols selected optimally from the symbol constellation diagram shows no significant change for the fixed transform methods. However, the PAPR for the multitransform-DSI method with just 1 dummy symbol is just $0.5 \mathrm{~dB}$ higher compared to the single carrier transmission. This difference can be further reduced to nearly 0 with an increase in the value of $N_{T}$ and the number of dummy symbols $N_{D}$.

The difference between the fixed transform and multitransform methods becomes even more pronounced when only a subset of the OFDM channels are included in the transform. For example, for the IEEE 802.11 standard with 64 OFDM channels, 11 of the OFDM channels carry zeros and 48 of the channels are used for data using a 48-point precoding transform. For this case, the simulation results show that for 64 QAM the multitransform-DSI method with $N_{T}=16$ and the number of dummy symbols $N_{D}=1$ provides a PAPR value of $5.4 \mathrm{~dB}$ that is reduced to about $5.0 \mathrm{~dB}$ with $N_{D}=2$ and is equal to that obtained for the single carrier transmission thus eliminating any PAPR penalty of the multicarrier OFDM system. In contrast to this, for exactly the same simulation conditions, the PAPR of the fixed transform methods is equal to about $9.2 \mathrm{~dB}$. Limiting the dummy symbol search to the optimal subset of 4 symbols of the constellation diagram does not result in any significant PAPR performance difference.

\section{Disclosure}

The material in this paper is protected under US Patent 8,995,542, March 31, 2015.

\section{Competing Interests}

The authors declare that there is no conflict of interests regarding the publication of this paper.

\section{References}

[1] R. Van Nee and R. Prasad, OFDM for Wireless Multimedia Communications, Artech House, Norwood, Mass, USA, 2000.

[2] J. R. Simic, "Analysis of OFDM multiuser system over fading channels," in Proceedings of the 5th International Conference on Telecommunications in Modern Satellite, Cable and Broadcasting Service (TELSIKS '01), Nis, Yugoslavia, September 2001.

[3] A. R. S. Bahai and B. R. Saltzberg, Multi-Carrier Digital Communications: Theory and Applications of OFDM, Kluwer Academic/Plenum Publishers, 1999.

[4] J. Heiskala and J. Terry, OFDM Wireless LANs: A Theoretical and Practical Guide, Sams Publishing, Indianapolis, Ind, USA, 2002.

[5] M. D. Benedetto and G. Giancola, Understanding Ultra Wide Band Radio Fundamentals, Prentice Hall, New York, NY, USA, 2004.

[6] IEEE Standard, "Part 11: wireless LAN Medium Access Control (MAC) and Physical Layer (PHY) specifications: high-speed physical layer in the 5 GHZ band," IEEE Standard 802.11a, 1999.

[7] R. Kumar and M. Khan, "Mitigation of multipath effects in broadband wireless systems using quantized state adaptive equalization method," in Proceedings of the IEEE Aerospace Engineering Conference, pp. 1-9, Big Sky, Mont, USA, March 2006.

[8] R. Kumar, "A higher-order analysis of the distortion effects of nonlinera amplifiers on CDMA signals," in Proceedings of the IEEE Aerospace Engineering Conference, pp. 1-10, Big Sky, Mont, USA, March 2008.

[9] R. Kumar, "Adaptive Compensation Systems for Mitigating Distortion due to Nonlinear Power Amplifiers," Patent Application US 2011/0234314, 2011.

[10] R. Kumar, "Systems and Methods for Mitigating Spectral Regrowth from Nonlinear Systems," Patent Application US 2012/ 0280749, November, 2012. 
[11] R. Kumar, "Multi Transform OFDM systems and Methods with Low Peak to Average Power Ratio Signals," U. S. Patent 8,995,542, March, 2015.

[12] L. Wang and C. Tellambura, "A simplified clipping and filtering technique for PAR reduction in OFDM systems," IEEE Signal Processing Letters, vol. 12, no. 6, pp. 453-456, 2005.

[13] R. W. Bäuml, R. F. H. Fischer, and J. B. Huber, "Reducing the peak-to-average power ratio of multicarrier modulation by selected mapping," Electronics Letters, vol. 32, no. 22, pp. 20562057, 1996.

[14] S. H. Müller and J. B. Huber, "OFDM with reduced peakto-average power ratio by optimum combination of partial transmit sequences," Electronics Letters, vol. 33, no. 5, pp. 368369, 1997.

[15] S. H. Muller and J. B. Huber, "A comparison of peak power reduction schemes for OFDM," in Proceedings of the IEEE Global Communication Conference (GLOBECOM '07), pp. 1-5, November 1997.

[16] H.-G. Ryu, J.-E. Lee, and J.-S. Park, “Dummy Sequence Insertion (DSI) for PAPR reduction in the OFDM communication system," IEEE Transactions on Consumer Electronics, vol. 50, no. 1, pp. 89-94, 2004.

[17] P. Van Eetvelt, G. Wade, and M. Tomlinson, "Peak to average power reduction for OFDM schemes by selective scrambling," Electronics Letters, vol. 32, no. 21, pp. 1963-1964, 1996.

[18] H. Ochiai and H. Imai, "MDPSK-OFDM with highly power efficient block codes for frequency-selective faing channels," IEEE Transactions on Vehicular Technology, vol. 49, no. 1, pp. 74-82, 2000.

[19] T. A. Wilkinson and A. E. Jones, "Minimization of the peak to mean envelope power ratio of multicarrier transmission schemes by block coding," in Proceedings of the IEEE 45th Vehicular Technology Conference, pp. 825-829, Chicago, Ill, USA, July 1995.

[20] A. E. Jones, T. A. Wilkinson, and S. K. Barton, "Block coding scheme for reduction of peak to mean envelope power ratio of multicarrier transmission schemes," IEE Electronic Letters, vol. 30, no. 25, pp. 2098-2099, 1994.

[21] I. Baig and V. Jeoti, "PAPR analysis of DHT-precoded OFDM system for M-QAM," in Proceedings of the International Conference on Intelligent and Advanced Systems (ICIAS '10), pp. 1-4, Kuala Lumpur, Malaysia, June 2010.

[22] V. Jeoti and I. Baig, "DCT precoded SLM technique for PAPR reduction in OFDM systems," in Proceedings of the International Conference on Intelligent and Advanced systems (ICIAS '10), pp. $1-6$, June 2010.

[23] S.-W. Kim, J.-K. Chung, and H.-G. Ryu, "PAPR reduction of the OFDM signal by the SLM-based WHT and DSI method," in Proceedings of the IEEE Region 10 Conference (TENCON '06), pp. 1-4, IEEE, Hong Kong, November 2006.

[24] R. Van Nee and A. De Wild, "Reducing the peak-to-average power ratio of OFDM," in Proceedings of the 48th IEEE Vehicular Technology Conference (VTC '98), vol. 3, pp. 2072-2076, Ottawa, Canada, May 1998.

[25] J.-C. Wu, C.-M. Li, and C.-C. Tseng, "A PDSI with STBC scheme for PAPR reduction in OFDM system," in Proceedings of the International Conference on Consumer Electronics, Communications and Networks (CECNet '11), pp. 3851-3854, IEEE, Xianning, China, April 2011.

[26] C. Oestges and B. Clerckx, MIMI Wireless Communications, Academic Press, 2007.
[27] A. B. Gershman and N. D. Sidiropoulos, Space-Time Processing for MIMO Communications, John Wiley \& Sons, 2005.

[28] L. L. Hanzo, Y. Akhtman, Li. Wang, and M. Jiang, MIMI-OFDM for LTE, WiFi and WiMAX, John Wiley \& Sons, New York, NY, USA, 2011.

[29] Y. Tadokoro and T. Higuchi, "Discrete Fourier transform computation via the Walsh transform," IEEE Transactions on Acoustics, Speech, and Signal Processing, vol. 26, no. 3, pp. 236240, 1978. 


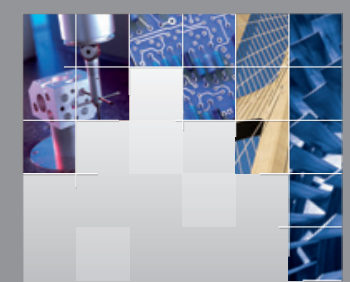

\section{Enfincering}
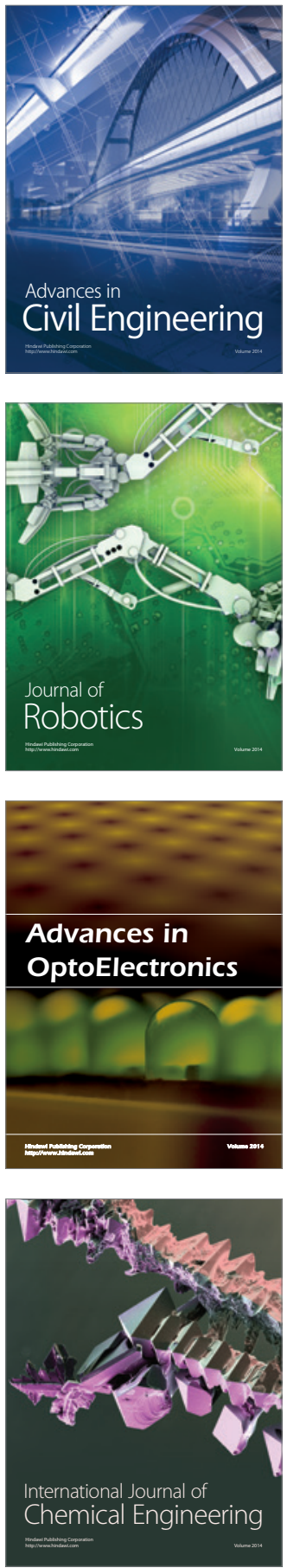

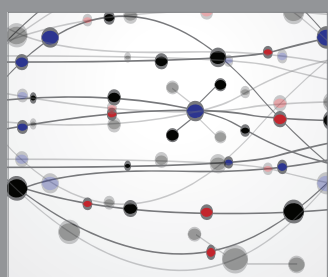

The Scientific World Journal

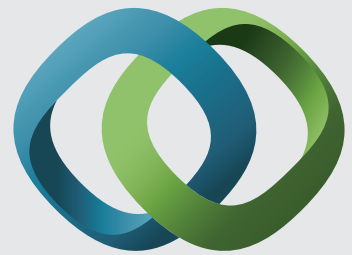

\section{Hindawi}

Submit your manuscripts at

https://www.hindawi.com
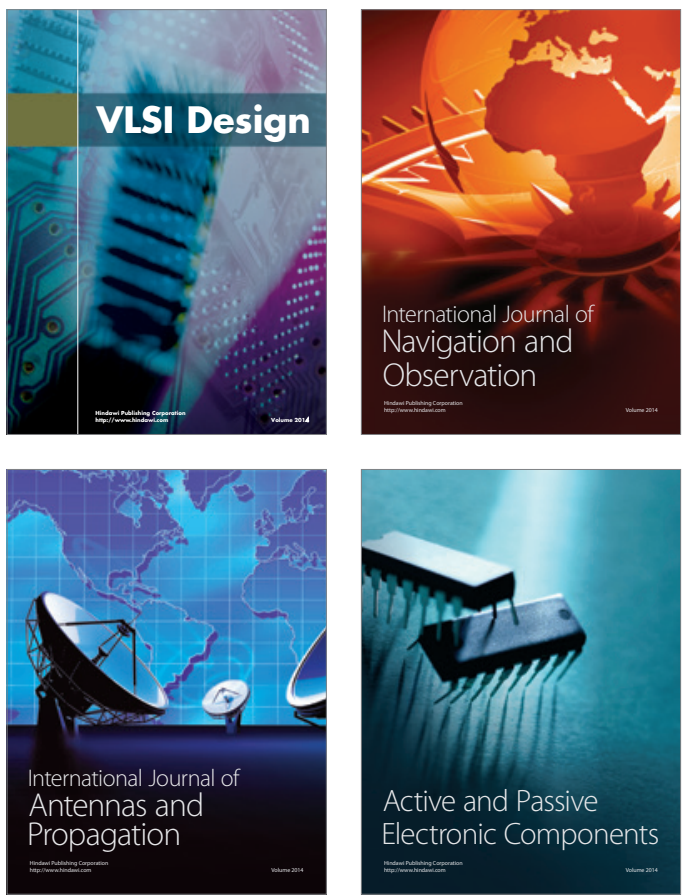
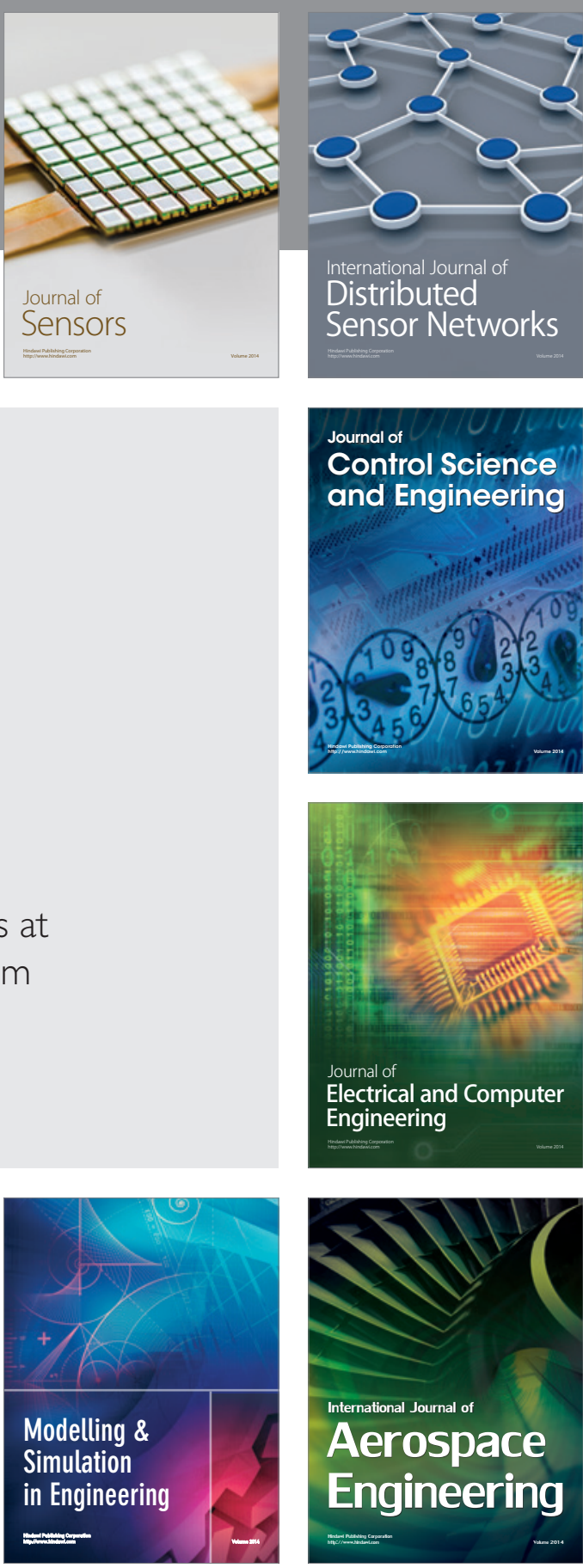

International Journal of

Distributed

Sensor Networks

$-$

Joumal of

Control Science

and Engineering
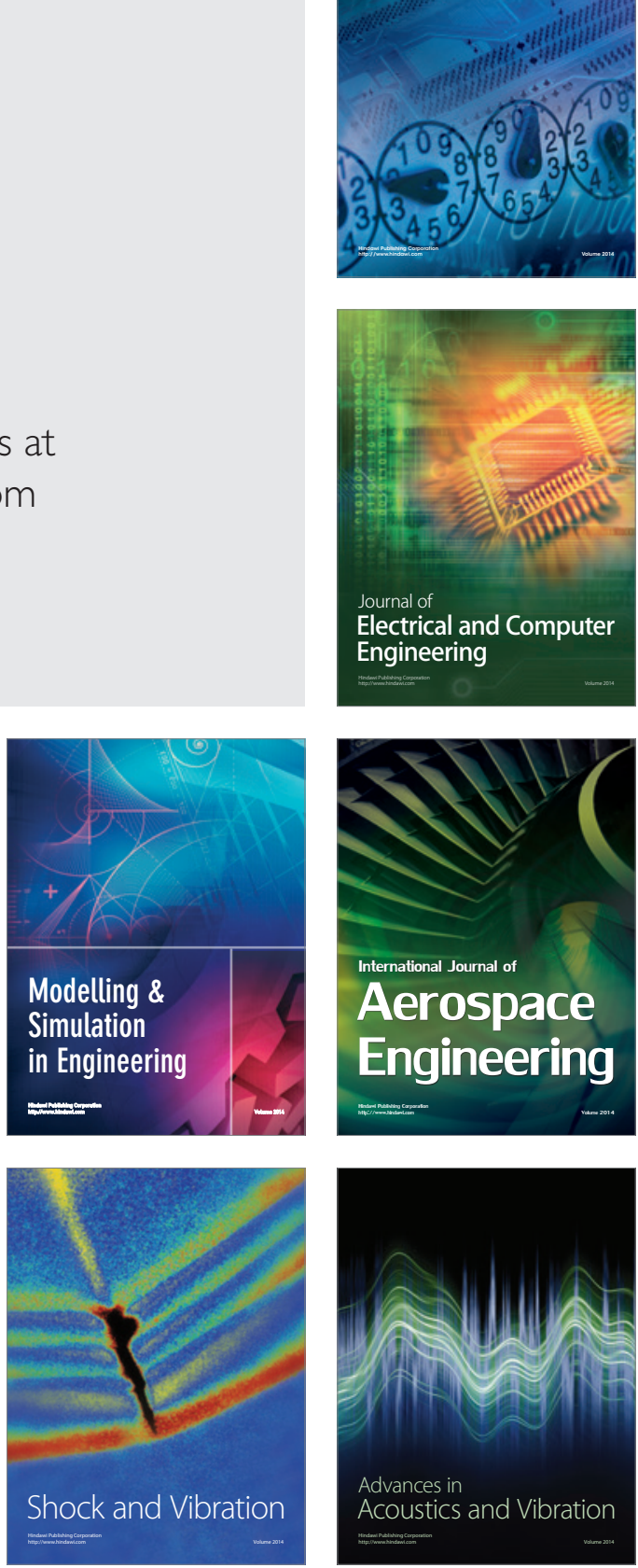